\title{
Die Entwicklungslogik der nominalen Determinativkomposition im Deutschen - verstanden als Grammatikalisierung
}

\author{
Martina Werner (Wien)
}

\begin{abstract}
Under the assumption of an individually grammatical semantics of word-formation types, this paper deals with the development of determinative compounding in the history of German from an inner-morphological perspective. Diachronically, the former research observed that German nouns have become "longer", i. e. more complex (firstly been formulated by Wurzel 1996). Additionally, some morphological restrictions within nominal compounding have been documented for historical stages of German, but some of them cannot be attested in presentday German. This suggests a formally and semantically motivated, inner-morphological change which can be described in terms of grammaticalization theory. For this purpose, previous results from historical word-formation and semantics will be combined with new observations on morphological discontinuities in the history of German, especially by focussing on morphological restrictions from a synchronic and a diachronic perspective. Since this approach comprises various linguistic phenomena (such as the diachronic development of part of speeches, (de-)referencialization, definiteness etc.) and since morphological restrictions have merely not been documented empirically - neither for present-day nor for historical stages of German, the paper aims at sketching the most prominent lines of development from a theoretical perspective, also by contrasting them with results from language typology, variational linguistics, and semantics. Additionally, the paper provides morpho-syntactical prospects for further theoretical and empirical research on historical morphology, also by conceiving word-internal language change as an integral part of the dynamics of morphosyntactic structuring.
\end{abstract}

\section{$1 \quad$ Ausgangspunkt und Orientierung}

Der vorliegende Beitrag ${ }^{1}$ beschäftigt sich mit der Frage, warum substantivische Determinativkomposita (im Folgenden kurz: Komposition) im Verlauf der deutschen Sprachgeschichte immer „länger“ (i. S. v. komplex) wurden (erstmals formuliert von Wurzel 1996). Daran schließt sich aus morphologischer Sicht die Frage an, welche wortinternen Veränderungen sich beobachten lassen. Wörter wie Fallschirmspringbenutzungsanweisung wird man in anderen Sprachen wie etwa dem Englischen schwerlich finden, und dies obwohl dort sowohl die

\footnotetext{
${ }^{1}$ Für Diskussionen bzw. muttersprachliche Beispiele danke ich Gábor Fonyad, Ljudmila Geist, Ingeborg Geyer, Katharina Hartmann, Gianina Iordăchioaia und Anthony Rowley.

Linguistik online 77, 3/16 - http://dx.doi.org/10.13092/lo.77.2908
}

CC by 3.0 
Komposition als auch die Derivation produktive Wortbildungsprozesse darstellen. Das Deutsche ist typologisch bekannt für seine langen Wörter. Ein kurzer Blick in historische Texte des Deutschen, etwa des Ahd. (wie teiga-trōc, hanen-fouz, sunnūn-tac, Bsp. aus Splett 2000: 1214) und Mhd. (wie bach-brunne, bat-stube, lant-bote, Bsp. aus Lexer 2011), lässt jedoch erkennen, dass morphologisch komplexe, d. h. mehrgliedrige Wörter, insbesondere mit (de)verbalen Konstituenten, wie das gerade erwähnte nicht zu finden sind. Damit liegt die Annahme eines Sprachwandels in der Geschichte des Deutschen nahe, dessen Motivation zwar aus syntaktischer (cf. z. B. Demske 2001), bislang nicht jedoch aus innermorphologischer, wortinterner Perspektive zu ergründen versucht wurde.

Die in dem vorliegenden Beitrag zu leistende Ermittlung möglicher, innermorphologischer Sprachwandelfaktoren in der Sprachgeschichte des Deutschen erfolgt unter Annahme einer diachron beobachtbaren Grammatikalisierung (im Sinne von Lehmann 1995) innerhalb des Wortbildungstyps der substantivischen Determinativkomposition. Strukturell der Determinativkomposition ähnlich, da ebenfalls rechtsköpfig, ist die synthetische Komposition (auch: Zusammenbildung, Rektionskomposition). Sie ist jedoch historisch von erster aus verschiedenen, synchronen wie diachronen Gründen abzugrenzen. Die in diesem Zusammenhang wichtigste Beobachtung dürfte dabei sein, dass sich die synthetische Komposition bereits im Ahd. belegen lässt, während dies für die Determinativkomposition zumindest strittig, wenn auch nicht ausgeschlossen ist (cf. hierzu Kapitel 2 und 5). Da mit der Annahme einer Grammatikalisierung von Wortbildungsstrukturen eine Fülle von ihrerseits teils kontroversen Phänomenen (wie der Definitheit, Nominalsemantik, Syntax, u. v. m.) verbunden ist, versteht sich der vorliegende Beitrag einerseits als Diskussionsgrundlage, andererseits als weiterer Schritt in die Richtung, historische Wortbildung unter grammatiktheoretischer Perspektive in den Blick zu nehmen. Inwieweit dem Erst- oder aber dem Zweitelement dabei eine entscheidende Rolle beim Aufbau grammatischer Semantik zukommt, widmet sich Abschnitt 3. Bisherige Ergebnisse der historischen Wortbildungsforschung sollen, soweit im Rahmen dieses Beitrags möglich, in die Axiomatik der Grammatikalisierung integriert und ggf. neu interpretiert sowie mögliche, hierdurch neu entstehende und ggf. empirisch zu erbringende Forschungsdesiderate aufgezeigt werden. Während Abschnitt 4 synchrone (gegenwartssprachliche) Verteilungen in den Blick nimmt, begibt sich Abschnitt 5 in die Dynamik der Diachronie. Eine Zusammenführung der diachronen und synchronen Befunde und daraus abzuleitender, formaler und semantischer Konsequenzen in Bezug auf Form und Semantik der Komposition wird in Abschnitt 6 herausgearbeitet. Zur Annäherung an das Paradigma der Grammatikalität bzw. Grammatikalisierung (Abschnitt 1.1) soll in Analogie zur Syntax der Betrachtungsschwerpunkt auf morphologisch belegbare wie nicht-belegbare Distributionen gelegt werden. Zu den damit verbundenen, methodologischen Prämissen cf. den darauffolgenden Abschnitt (1.2).

\subsection{Warum Grammatikalisierung?}

Die Konzeption, sprachstufenspezifische Generalisierungen aus synchronen Distributionsbeschränkungen, d. h. belegbaren und nicht belegbaren Wortbildungsmustern, abzuleiten und diese diachron unter der Perspektive der Grammatikalisierung miteinander in Beziehung zu setzen, um daraus Konsequenzen für die morphologische Strukturbildung abzuleiten, blieb für das Deutsche im Bereich der Komposition bislang mehrheitlich ungenutzt, obgleich für ande- 
re Wortbildungstypen (wie etwa die Suffigierung, cf. hierzu z. B. Leiss 2005; zur Suffixoidbildung cf. Zifonun 2012) Ansätze zu einer grammatischen Beschreibung (hier: der Quantifizierung) vorliegen bzw. bereits früh etwa für die Apposition Ähnliches hervorgehoben wurde (cf. z. B. Löbel 1986). Auch im Rahmen syntaxtheoretischer Beiträge wird die Bedeutung morphosyntaktischer Gesetzmäßigkeiten für die Komposition betont (cf. bspw. Alexiadou/Iordăchioaia 2015); so auch für die Diachronie (cf. z. B. Demske 2001). Daraus lässt sich die Frage ableiten, ob sich auf Basis der bisherigen Beobachtungen also GesetzmäBigkeiten ermitteln lassen, die die Annahme einer Grammatikalisierung wahrscheinlich machen sowie welcher semantische Inhalt grammatikalisiert wird.

Zur Diachronie der Komposition ist zunächst bekannt, dass aus syntaktischen Strukturen (cf. Givón 1971) bzw. syntaktischen (Vor-)bedingungen (cf. Gaeta 2008), genauer, nicht unter VO-, sondern nur unter OV-konfigurationellen Bedingungen (Haider 2001, 2015) die Komposition entstehen kann. Komplexe Komposita sind damit niemals linksköpfig. Die Komposition mündet entwicklungsgeschichtlich und übereinzelsprachlich überaus häufig in Derivation und diese wiederum mündet überaus häufig in Flexion (cf. z. B. die Partizipialbildung des Deutschen oder die Diminution), die übereinzelsprachlich häufiger als Suffixe als als Präfixe realisiert werden (sog. suffixing preference, cf. Cutler et al. 1985). Die Domäne der Komposition als ein Glied dieser Entwicklungskette wird über die Funktion zur Spezifikation gefasst (cf. Kapitel 2), was aus inhaltlicher Sicht allerdings die Frage nach einer inhärenten Semantik der synthetischen (im Gegensatz zur analytischen) Form und aus morphologischer Sicht mögliche, formale Restriktionen der Komposition (cf. Kapitel 4 zur Snychronie, Kapitel 5 zur Diachronie) unberücksichtigt lässt. Daher soll in diesem Beitrag vor dem Hintergrund morphologischer Theorie(bildung) der Fokus auf wortinternen Restriktionen und deren (möglichem) Abbau liegen, da sich diachron motivierte Diskontinuitäten sowie synchrone Restriktionen im Bereich der Wortarten und beteiligter Wortbildungstypen (v. a. der Derivation) beobachten lassen, die vor dem Hintergrund des eingangs festgestellten, morphologischen Komplexitätszuwachses die Annahme einer Grammatikalisierung rechtfertigen. Der vorliegende Beitrag versteht sich somit als weiterer Schritt in die Richtung, historische Wortbildung als grammatische Strukturbildung auch semantisch ernst zu nehmen und synchrone Distributionsrestriktionen vor dem Hintergrund von motivierter Musterbildung der Diachronie aus sich heraus ,erklärbar” zu machen.

\subsection{Methodologischer Rahmen}

Da eine umfassende, d. h. empirisch gestützte, diachrone Arbeit zur Entstehung der Komposition unter Zuhilfenahme des Sprachvergleichs mit anderen germanischen Sprachen und ihrer jeweiligen Varietäten bislang ein Forschungsdesiderat bleiben muss, stützt sich der vorliegende Beitrag auf bisherige Befunde der Forschungsliteratur, die unter dem Aspekt der Grammatikalisierung betrachtet und ggf. neu interpretiert werden sollen. Um darüber hinaus mögliche, empirische Beleglücken zu füllen, wurde für historisches Sprachmaterial das Wörterbuch von Lexer (2011) zum Mhd. bzw. das Ahd. Wörterbuch von Splett (1993) verwendet, aus denen die im Folgenden zitierten Belege, so nicht anders angegeben, stammen. Für gegenwartssprachliches Material wurden zur Ermittlung von Stichproben des Deutschen das DWDS- 
Korpus (cf. dwds.de) sowie das Austrian Media Corpus (cf. www.oeaw.ac.at/acdh/en/amc [19.05.2016]) beforscht.

Da in der Gegenwartssprache im Gegensatz zu früheren Sprachstufen Sprecherurteile zu Belegen verfügbar sind, die (Nicht-)Akzeptabilität prüfen können, ist es aus synchroner Sicht methodologisch aussichtsreich, diese zur Erfassung gegenwartssprachlicher Strukturen und der sich daraus ergebenden Systematizität als deskriptive Generalisierungen in die Theoriebildung miteinzubeziehen. Diachron ist (lediglich) die Möglichkeit zu Korpusuntersuchungen gegeben. Da jedoch bislang keine morphologisch detailliert annotierten historischen Korpora zum Deutschen vorliegen, kann hier nur stichprobenartig und anhand dessen, was sprachstufenspezifisch und übereinzelsprachlich in der Literatur beschrieben wurde, bei der vorliegenden Betrachtung Berücksichtigung finden. Da also axiomatisch statt motivierter Einzelfallbetrachtung ( $>$ Lexikalisierung) einer morphosyntaktisch-regelbasierten, reihenbildenden Systematizität ( $>$ Grammatikalisierung) gefolgt wird, sollen mithilfe von Distributionsproben synchrone Akzeptabilitätsurteile validiert, auf Basis der so erzielten, deskriptiven Beobachtungen generalisiert und schließlich mit Beobachtungen aus der Diachronie in Beziehung gesetzt werden. ${ }^{2}$

Morphologische Produktivität ist damit in der Diachronie validierbar über sprachstufenspezifische Innovationen und sprachstufenspezifischen Neuerungen, wobei hier, soweit möglich, die Lemmata aus bereits zuvor belegten Sprachstufen auszuschließen sind. Produktiv im synchronen Sinne ist also eine Eigenschaft, die dann vorliegt, wenn ein Wortbildungstyp uneingeschränkt reihenbildend ist bzw. seine Einschränkung wortartspezifischen, niemals aber einzelfall-basierten Charakter hat. Scherzbildungen (wie bspw. Toll-ität) sind auszuschließen (cf. hierzu Bauer 2001). Die Unterscheidung zwischen Grammatikalisierung und Lexikalisierung ist morphologisch zentral, da Lexikalisierungen ihr morphosyntaktisches Verhalten grundlegend verändern können. Ein Beispiel für grammatikalisierte Nominalisierungen wären substantivierte Infinitive, welche im Gegenwartsdeutschen nicht pluralisierbar sind (wie das Rudern - *die Rudern, das Schreien - *die Schreien, das Herumgehen - *die Herumgehen, cf. z. B. Ehrich 1991). Lexikalisierungen jedoch widersetzen sich häufig dieser Regelhaftigkeit (wie bspw. das Essen - die (verschiedenen) Essen, das Leben - die Leben). Das ursprünglich grammatische Potenzial der Bildungssystematizität der substantivierten Infinitive wird hier

\footnotetext{
${ }^{2}$ Parallel zu den erhobenen Daten müsste methodologisch in künftigen, morphologischen Untersuchungensozusagen als „Vergleichsfolie“ - ebenso erhoben und offen gelegt werden, was nicht zu erheben war (sprachstufenspezifische Restriktionen), da das nicht Erhebbare zur morphologischen Theoriebildung ebenso wichtig ist wie das Erhebbare. Natürlich stellen frühere Sprachstufen aufgrund des nicht uneingeschränkt verfügbaren Sprachmaterials eine solche Empirie wie Theorie vor Herausforderungen. Allerdings lassen sich u. U. auch aus niedriger frequenten, systematischen Phänomenen Rückschlüsse auf die Verbreitungshäufigkeit ziehen, nur bedingt aber auf die Produktivität des jeweils produktiv operierenden Systems, welches am besten durch die Adhoc-Bildungen repräsentiert wird. Daraus ergibt sich, dass Nicht-Belegbares ebenso wie Belegbares Repräsentativität genießen darf. Somit sind für künftige, empirische Forschung absolute Beleganteile vorrangig zu berücksichtigen sowie relative Beleganteile nur im Hinblick auf sprachstufenspezifische Verschiebungen (was war „,vorher“?/was war „nachher“?) methodologisch in den Betrachtungsvordergrund zu rücken. Eine weitere Achse der Validierung ergäbe sich zuletzt aus der Verfügbarkeit verwandter und nicht-verwandter Sprachen (sprachtypologische Ebene) sowie aus der sprachlichen Variation, so dass einzel- bzw. regionalsprachliche Parameter mitberücksichtigt werden können.
} 
punktuell, aufgrund fehlender Reihenbildung der Lexikalisierungen allerdings nicht systematisch neutralisiert.

Neben den formal-grammatischen Gesichtspunkten können Lexikalisierungen auch über ihre Bedeutung teilweise identifiziert werden. So sind im Gegenwartsdeutschen produktiv gebildete Ableitungen des Suffixes -tum ausschließlich mit Personenbezeichnungen möglich, cf. Studententum, Matrosentum, Professorentum, was morphologisch, da synchron nicht mehr alle Wortarten, sondern nur noch Personenbezeichnungen mit diesem Suffix nominalisierbar sind, bereits einer starken formalen Einschränkung gleichkommt. Die Bedeutung der Bildung ist ein Personenabstraktum ,Art, Stand von Studenten/Matrosen/Professoren'. Eine Bildung wie Herzogtum dagegen fügt sich zunächst in dieses Muster, ist aber aufgrund seiner davon unterscheidbaren Bedeutung als Lexikalisierung einzustufen (,Reich, Land eines Herzogs', nicht ,Art, Stand eines Herzogs'). Auch formal ist dies an dem Fehlen der Fuge zu erkennen (*Herzögetum) sowie an der Pluralfähigkeit, die produktiven tum-Suffigierungen aufgrund des ererbten kontinuativen Genus neutrum (cf. z. B. Balles 2004 zum Idg.) verwehrt bleibt (*Professorentümer, *Studententümer). Auf Basis der formalen wie semantischen Abweichungen können durch die geschilderten Verfahren einzelne Lexikalisierungen identifiziert werden, obgleich bei Komposita innerhalb desselben Erst- bzw. Zweitelements auch unterschiedliche Formen von Lexikalisierung zugrunde liegen können wie bei Sägeblatt, Blatt der Säge' im Gegensatz zu Sägespäne ,Späne, die durch das Sägen entstehen' vs. ?,Späne, die bei der Säge sind'. Morphologisch ergibt sich daher bei der Diskussion von Beispielen die Konsequenz, dass einzelne Beispiele, solange sie in ihrer Natur nicht reihenbildend und damit systematisch sind, niemals geeignet sein können, die Aufstellung einer Wortbildungsregel bzw. -systematizität in Frage zu stellen, während umgekehrt morphologisch systematische Reihen als auch quantitativ repräsentative (Gegen-)Beispiele in der morphologischen Diskussion ernst zu nehmen sind.

\section{Zum Phänomen der Komposition}

Als Funktion der Determinativkomposition wird traditionell die Funktion der Spezifikation angeführt. Die Konstituenten von Nomina wie Semmelknödel, Kartoffelknödel und Speckknödel spezifizieren also (in Form des jeweiligen Erstelements) den morphologischen Kopf Knödel. Mit der traditionellen Definition der Komposition über Spezifikation ist aus innermorphologischer Sicht die Schwierigkeit verbunden, dass sich damit keine synchronen sowie diachronen Distributionsbeschränkungen erklären lassen. So stellt sich bspw. die Frage, warum derivierte Adjektive ebenso wie die Partizipia I und II und einige weitere, über Derivation markierte Konstituenten nicht als Erstelemente von Komposita realisiert werden können (cf. *Künstlichfaser, *Gewebtteppich). Die Funktion der Spezifikation soll daher im folgenden Abschnitt vor der im vorherigen Abschnitt zugrundegelegten Axiomatik diskutiert werden, während die formale Abgrenzung der Determinativkomposition zur synthetischen Komposition in Abschnitt 2.2 eingehender betrachtet werden soll. 


\subsection{Morphosyntaktische Äquivalente zur Komposition}

Als morphologisches Pendant zur Komposition vom Typ Jugendgeist unter der Annahme von Spezifikation gelten im Allgemeinen die Adjektivattribute (Typ jugendlicher Geist). Da es sich um morphosyntaktische Äquivalente handelt, sollte eine Betrachtung der Grammatikalisierung der Komposition eigentlich die Frage mitbeantworten können, warum das Deutsche nicht attributive Adjektive anstelle der Komposition grammatikalisiert hat bzw., da beide Muster im Deutschen existieren, eine funktionale Differenzierung auf synchroner oder diachroner Ebene zu beobachten ist. Schuster (2016) zufolge lässt sich diese Frage diachron nicht eindeutig beantworten, da diesbzgl. ein Zusammenspiel aus sprachinternen und externen Sprachwandelfaktoren anzunehmen ist (cf. auch Abschnitt 4.1). Ein Grund könnte allerdings u. U. die bislang unzureichende Forschungslage zu diachronen Restriktionen der Komposition sein, und zwar nicht nur in Bezug auf die Standardsprache, sondern ebenso in Bezug auf die Varietäten des Deutschen. Während in den letzten Jahren verstärkt ein Interesse an flexionsmorphologischen Fragen in den Varietäten zu beobachten ist, dürfte die Wortbildungslehre in den Varietäten des Deutschen gegenwärtig das am stärksten vernachlässigte Gebiet der Dialektmorphologie sein. Dies gilt umso mehr für historische Sprachstufen. Der vorliegende Beitrag versucht neben der Nachzeichnung einer möglichen standardsprachlichen Entwicklung daher, soweit möglich, auch Anknüpfungen an dialektale Varietäten herzustellen, auch, um Forschungsanstöße für die Dialektologie zu geben. Der vorliegende Beitrag versteht sich darüber hinaus auch als weiterer Schritt zur Erhellung der Frage, inwieweit zwischen Adjektivattributen und Komposition in der Diachronie des Deutschen Konkurrenzverhältnisse bestanden.

$\mathrm{Da}$ im vorliegenden Beitrag jedoch die Spurensuche einer (möglichen) Grammatikalisierung aufgenommen werden soll, muss vor allem die Frage nach einer für die Komposition spezifischen grammatischen Semantik in den Betrachtungsfokus gerückt werden. Damit kann gleichzeitig an sprachtypologische Erwägungen für weitere Forschung insofern angeknüpft werden, als dass der Wortbildungstyp Komposition nicht in allen Sprachen zwar ggf. dokumentiert (über Lehnübersetzungen oder Entlehnungen), aber nicht produktiv (im Sinne von reihenbildend mit Erstelementen verschiedener Wortarten) ist. Als eine Sprache ohne produktive Determinativkomposition gilt gemeinhin das Russische. ${ }^{3}$ Dort werden anstelle von Komposita des Deutschen entsprechende analytische Konstruktionen gebildet, vornehmlich mit Adjektivattributen wie bspw. ptič"'e penie wörtlich ,vögelischer Gesang', d .h. ,Vogelgesang' oder akonni kasjak wörtlich ,fensterlicher Pfosten', d. h. ,Fensterpfosten”. Die gemeinsame Vergleichsebene zwischen Sprachen mit und ohne Komposition stellt daher neben formalen Gesichtspunkten die Semantik dar (cf. Kapitel 4). Vor der Feststellung eines grammatischen Inhalts jedoch steht die Frage, wo eine solche ggf. zu beobachten ist: im Erstelement (cf. Abschnitt 3.1) oder im Zweitelement (cf. Abschnitt 3.2).

\footnotetext{
${ }^{3}$ Zwar sind im Russischen seltene Fälle von nominalen Determinativkomposita dokumentierbar, da jedoch die meisten Fälle auf Entlehnungen oder Lehnübersetzungen rückführbar sind und da andere Formen wie V+NKomposita i. d. R. nicht feststellbar sind, wird die Einordnung als Sprache ohne Determinativkomposition davon nicht berührt und kann somit bestehen bleiben. Allerdings gibt es die synthetische Komposition, die in formaler Analogie zum Deutschen auch als Suffigierung mit komplexer Derivationsbasis gefasst werden kann, wie bspw. im Russischen wie $l e d_{\mathrm{N}}-O_{\mathrm{FE}}-k o l_{\mathrm{N}}$,Eis-Brecher' oder $z v u k_{\mathrm{N}}-O_{\mathrm{FE}}-z a p i s_{\mathrm{N}}$, Ton-Aufnahme‘.
} 
Damit wären zunächst die konkreten Prinzipien der Strukturbildung in den Fokus der Grammatikalität gerückt - was gleichzeitig die Suche nach fehlender Akzeptabilität bzw. Grammatikalität, d. h. Distributionsbeschränkungen und semantischem Komplexitätszuwachs impliziert. Bevor die diachrone Seite der Komposition (Abschnitt 5) und die Frage nach einer möglichen grammatischen Funktion (Abschnitt 4) beleuchtet werden, ist es zunächst notwendig, den Bereich der Komposition formal abzustecken (Abschnitt 3). Im Bereich der Komposition gibt es zunächst zwei Typen, die in einigen Forschungsansätzen zusammengefasst werden: Determinativkomposition und synthetische Komposition (auch: Zusammenbildung, Rektionskomposition). Der Frage ihrer gegenseitigen Abgrenzbarkeit widmet sich der nächste Abschnitt.

\subsection{Zwei Arten von Komposition und typologische Einordnung}

Neben die Determinativkomposition (wie bspw. N+N Haustür oder V+N Turnhose) wird die synthetische Komposition gestellt, welche sich von ersterer primär durch das Aufweisen eines deverbalen Kopfes unterscheidet (wie bspw. Wetterbeobachtung). Die Trennung zwischen beiden Kompositatypen ist kontrovers, v. a. aufgrund der Ambiguität der synthetischen Komposition, strukturell zwischen Derivation und Komposition verortet zu sein: So ist die Bildung Wetterbeobachtung auf zwei Arten analysierbar: entweder als $\left[\left[\text { Wetter }_{\mathrm{N}}\right]\left[\text { Beobachtung }_{\mathrm{N}}\right]\right]_{\mathrm{N}}$ und damit als Determinativkomposition oder aber als [[[Wetter $\left.{ }_{\mathrm{N}}\right]$ beobacht-v]-ung $\left.]\right]_{\mathrm{N}}$ und damit als synthetische Komposition. Demzufolge wäre im ersten Fall die Paraphrase ,Beobachtung des Wetters', im zweiten Fall hingegen, Vorgang/Situation, bei dem/der das Wetter beobachtet wird' anzugeben.

Neben der synchronen strukturellen Analyse (zu Details cf. der Sammelband von Alexiadou/Rathert 2010; Ackema/Neeleman 2004; McIntyre 2015 u. v. a.) lassen sich auch diachron Argumente für eine Unterscheidung beider Kompositionstypen finden: So ist die Datierung der Determinativkomposition, nicht aber der synthetischen Komposition zum gegenwärtigen Zeitpunkt umstritten: Während sich bereits bei einer groben Sichtung des Sprachmaterials des ahd. Wörterbuchs (z. B. von Splett 1993) zeigt, dass das Ahd. bereits über synthetische Komposita verfügte (wie bspw. man-slahta ,Mord', wörtl. ,Menschenschlachtung', cf. Wilmanns 1893: 292), sind erste Belege von Determinativkomposita für das Ahd. fraglich, werden aber von einigen Forschern als solche angenommen (cf. bspw. Osthoff 1878; Carr 1939), während anderen Ansätzen zufolge die Herausbildung der Determinativkomposition erst später, d. h. erst im Fnhd. beobachtbar zu sein scheint (cf. z. B. Demske 2001, Wanzeck 2003). Da aus diachroner Sicht in jedem Fall das Gegenwartsdeutsche ${ }^{4}$ die längsten (hier im Sinne

\footnotetext{
${ }^{4}$ Auch für die Dialekte des Deutschen muss die Frage nach der synchronen Produktivität der Komposition und möglichen Realisierungsalternativen vorerst offen bleiben. Einerseits sind in den Dialekten des Deutschen, so bspw. im Bairischen, zahlreiche Bildungen belegbar (wie bair. Steckerlfisch ,Stöckchenfisch", Schneizdiachi ,Schneuztuch, Taschentuch"), andererseits sind ad hoc-Bildungen mit generischer Semantik jedoch häufig mündlich schwer zu belegen bzw. empirisch schwer zu elizitieren. Es handelt sich nach eigenen Stichproben m. E. in vielen Fällen um einen am Standard orientierten Hyperkorrektivismus, bei dem Komposita aus dem Standard in den Dialekt „entlehnt“ werden. Dies zeigt sich bspw. an der häufig fehlenden Akzeptabilität von ,echten" im Dialekt gebildeten ad-hoc-Komposita wie bspw. bair. *Kunsthonig (letzteres würde im Bairischen nach Auskunft von Muttersprachlern analytisch realisiert im Sinne von künstlicher Honig), standarddt. Küchenschrank, jedoch bair. Kastn in der Kuchl vs. ??Kuchlkastn; ebenso standarddt. Hemdtasche, bair. ??Hemad-
} 
von multilexikalisch) Komposita hat, muss es historisch zu Umkodierungen bzw. Komplexitätszuwächsen im Bereich der Determinativkomposition gekommen sein, was ebenfalls als Indiz für die Unabhängigkeit beider Wortbildungsmuster gewertet werden kann. Zudem lässt sich auch im kindlichen Spracherwerb des Deutschen eine Ausdifferenzierung zwischen Determinativ- und synthetischer Komposition im Verlauf der Sprachentwicklung feststellen (cf. Korecky-Kröll 2011).

Damit die aus der strukturellen Ambiguität der synthetischen Komposition sich ergebenden Implikationen nicht ein verzerrender Charakter für die Beschreibung der Determinativkomposition zukommt, werden im Folgenden Kompositionsbildungen mit deverbalen Köpfen von der Betrachtung ausgeschlossen, sofern es auf formaler Ebene keine Hinweise zugunsten einer Klassifikation als „echter” Determinativkomposition (z. B. durch Fugenelemente 5 , o. Ä.) gibt. Gerade in Bezug auf verschiedene „Interfigierungspräferenzen” zeigen sich nämlich einige distributionelle Unterschiede zwischen beiden Kompositionstypen, die geeignet sind, als diagnostische Kriterien bei der morphologischen Klassifikation mitberücksichtigt zu werden. So treten uneingeschränkt produktive Nominalisierungen im Gegenwartsdeutschen mit femininem Genus als Erstelemente von Determinativkomposita ausschließlich mit dem Fugenelement -s- auf (cf. auch Abschnitt 4.1, 5.3 und 6), cf. Schön-heit-s-operation oder Umgehung-sstraße. Die Interfigierung ist obligatorisch, d. h. nicht weglassbar ( ${ }^{*}$ Schönheitoperation, *Umgehungstraße). Bei synthetischen Komposita hingegen kann das Pluralmorphem des zugrundeliegenden Arguments (wie Schönheiten) der VP (z. B. Schönheiten malen) auch in der Komposition erhalten bleiben wie bei Schönheit-en-Maler. Die inhärent generische Semantik des Abstraktums wird in diesem Fall durch die Pluralisierung auf konkrete Vorkommnisse seiner selbst semantisch reduziert (Schönheiten im Sinne von ,schöne Menschen, schöne Frauen/Männer'). Umgekehrt, so könnte man sagen, verhindert das Fugen-s- der Determinativkomposita die Interpretation des Erstelements als internes Argument, so dass die entsprechende Bildung als synthetische Komposition zu analysieren ist. Das Fugenelement gewährleistet somit den Erhalt der abstrakten Semantik der Nominalisierung und gleichzeitig den Erhalt der Struktur des Determinativkompositums.

Auf Basis dieser Beobachtung dürften sich die meisten der synchronen Fugenelementschwankungen zu Komposita mit deverbalen Köpfen erklären lassen: Das Vorhandensein des Fugen$s$ (wie bei der Determinativkomposition) bzw. auf den Numerus rückführbare Phänomene (wie bei der synthetischen Komposition) lassen auf die jeweilige Geschichtetheit der zugrundeliegenden morphologischen Prozesse schließen, was bspw. das Minimalpaar (a) richtungsweisend bzw. (b) richtungweisend illustriert. In (a) liegt auf erster Analyseebene eine Determinativkomposition, in (b) Flexion (Partizip-I-Bildung) vor, während auf der zweiten Analyseebene bei (a) Derivation (Partizip-I-Bildung) und bei (b) $\mathrm{V}^{0}$ Richtung weisen zugrunde liegt:

\footnotetext{
/??Pfoat-taschn. Gerade aufgrund der problematischen Datenlage wäre daher eine systematische Erhebung bzw. Erschließung möglicher dialektaler bzw. regionaler Unterschiede für das gesamte deutschsprachige Gebiet und im innergermanischen Vergleich von wichtiger Bedeutung für die künftige Wortbildungsforschung, um ggf. mögliche Enkodierungsalternativen und Produktivitätsvariationen zu ermitteln.

${ }^{5}$ Die Termini Interfix bzw. Interfigierung und Fugenelement (Abk. FE) bzw. Fugenelementbildung werden in diesem Beitrag synonym verwendet.
} 
(a)

(b)
$=1$. Determinativkomposition,

2. Partizipialbildung

$=1$. Partizipialbildung,

2. $\mathrm{V}^{0}$ mit Argumentstruktur

Übertragen auf alle ggf. vermeintlichen Fugenelementschwankungen des Gegenwartsdeutschen von Bildungen mit einem deverbalen Kopf bedeutet dies, dass diese nochmals auf eine diesbezügliche Geschichtetheit, d. h. morphologische Motivation, untersucht werden müssen. Vor dem dargestellten Hintergrund würden sich die Schwankungen nicht unbedingt nur als Formen sprachlicher Variation, sondern vermutlich meist als Fälle einer grammatisch motivierten, strukturellen Opposition erweisen: die Komposition mit produktiv suffigierten Erstelementen, die, durch Derivation mittels Abstraktsuffix (wie -ung) als Femininum markiert, obligatorisch ein Fugen-s- zu sich nehmen (wie in richt-ung-s-weisend); die synthetische Komposition mit deverbalem Kopf, deren Erstelement, wenn deriviert, nie ein Fugenelement, sondern allenfalls ein Flexiv (wie das des Plurals) zu sich nimmt (wie in richt-ungweisend bzw. richtung-en-weisend). Die Kategorie Kasus ist durch die strukturelle Konfiguration der Konstituenten (Rechtsköpfigkeit) bereits implizit angelegt und damit morphologisch, weder bei der Determinativkomposition, noch bei der synthetischen Komposition, zu realisieren, cf. *Kindernhelfer, * Ärtenglauber, *Kindernfolger (cf. Rivet 1999; Steinbach 2002: 245). Somit eignet sich diese Kategorie nicht zur synchronen Abgrenzbarkeit beider Wortbildungsprozesse.

Ein mögliches Beispiel hingegen für eine implizite Mischung beider Kompositionsarten im Deutschen wäre etwa Gebrauchtwagen oder Gemischtwaren. Da Partizipia I herkömmlich nicht als Erstelemente von Determinativkomposita realisierbar sind (cf. *Wachendmann, *Platzend-/*Geplatzt-patrone), kann man vermuten, dass es sich bei der Bildung um die Kürzung eines einstigen synthetischen Kompositums mit phrasalem Kopf handelt, dessen deverbaler Kopf getilgt wurde (nämlich Gebrauchtwagenhändler oder -handel), dessen Erstelement nach Kürzung des nominalisierten Kopfes produktiv wurde (so auch bei Gemischtwaren zu Gemischtwarenhändler zur komplexen VP mit gemischten Waren handeln). Wie eigene Stichproben ergaben, sind solche Tilgungsprozesse jedoch i. d. R. für das Erstelement nicht musterbildend, sondern einzelfallspezifisch, was darauf hindeutet, dass beide Kompositionstypen (Determinativkomposition vs. synthetische Komposition) strikt voneinander getrennt d. h. grammatikalisiert sind. Dafür spricht auch, dass für das Ahd. bereits synthetische Komposita im Gegensatz zu Determinativkomposita (cf. Carr 1939) als gesichert gelten (cf. bereits Wilmanns 1896). ${ }^{6}$

Wie gezeigt wurde, gibt es übereinzelsprachlich Evidenz für die Trennung beider Wortbildungsprozesse und auch im Deutschen halten sich (vermeintliche) Kreuzungsprozesse beider

\footnotetext{
${ }^{6}$ Die Faustregel „Wann auch immer du einen Beleg als synthetisches Kompositum analysieren kannst, tue dies“ dürfte insofern gerechtfertigt erscheinen, als dass synthetische Komposita ebenso wie Konversionen primär syntaktisch komponiert sind (auch als sog. early compounding bezeichnet, cf. McIntyre 2015: 4) und weniger morphologischen Restriktionen unterliegen als primär morphologisch komponierte Determinativkomposita (sog. late compounding). Vor dem Hintergrund der Fraglichkeit von Kreuzungsprozessen wären mutmaßliche Kreuzungen beider Kompositionstypen (cf. auch Abschnitt 4.1) im Rahmen einer eigenen Studie für das Deutsche nicht nur quantitativ spannend zu untersuchen.
}

ISSN 1615-3014 
Wortbildungstypen in Grenzen, d. h. es können schwerlich Beispiele dafür in gegenwartssprachlichen Korpora gefunden werden (cf. vorheriger Abschnitt). Im Folgenden soll nun der Ort der Grammatikalisierung der Determinativkomposition (Erst- vs. Zweitelement) genauer verortet werden.

\section{Verortung der Grammatikalisierung}

Mit der Annahme einer Grammatikalisierung ist die Frage aufgeworfen, auf welche Art und wo eine wortinterne Grammatikalisierung stattfinden kann und welche Verbindungen sich in Bezug auf bislang dokumentierte Befunde aus der Sprachgeschichte in Bezug auf die Determinativkomposition ergeben. Der grammatische Beitrag sollte also einerseits strukturellen Gesetzmäßigkeiten (syntaktischer Aspekt), andererseits Wortarten und der in den grammatischen Kategorien zugrundeliegenden Semantik unterliegen. Rein formal wären dabei in Bezug auf die binäre Struktur der Komposition entweder das Zweit- (wie in Abschnitt 3.1) oder das Erstelement (cf. Abschnitt 3.2) in den Blick zu nehmen und auf in der Literatur beschriebene Sprachwandelphänomene abzubilden.

\subsection{Grammatischer Beitrag des Zweitelements?}

Der Kopf (Determinatum/Zweitelement) bestimmt bekanntlich die morphosyntaktischen Eigenschaften des gesamten Kompositums, so bspw. Tür (N.FEM.SG.) das Kompositum Haustür (N.FEM.SG.) usw., daraus ergibt sich jedoch die Frage, welche grammatisch ,verwertbaren" Formmerkmale sich in diesem Zusammenhang finden lassen. Wie bereits im vorherigen Abschnitt angedeutet, ist aus der Syntax bereits bekannt, dass komplexe Komposita niemals kopf-initial (linksköpfig) sein können, was mit OV-Serialisierung in Verbindung gebracht wurde (cf. Haider 2001, 2015). Die Syntax stellt damit sozusagen die (Vor-)Bedingungen für morphologische Komplexifikation bereit, die beide Konstituenten strukturell konfiguriert. Davon unabhängig (aber selbstverständlich bezogen darauf) ist die Frage nach einer grammatischen Funktion des Kopfes.

Für die Suffixoidbildung und Pseudokomposition ist für den substantivischen Bereich bereits der Nachweis erbracht worden, dass diese der Quantifizierung und damit der Beseitigung von sog. „Flexionslücken“ dienen (cf. Leiss 2005; Zifonun 2012): Nicht-pluralisierbare Substantive (wie bspw. Regen) erhalten durch entsprechende Wortbildungsformen einen Plural„Ersatz“ wie bspw. in Regen-fälle/-güsse. Ebenso können singularlose Substantive, i. d. R. Transnumeralia, typischerweise (cf. Vogel 1996) realisiert in Konversionen wie bspw. (das) Blau hierdurch quantifiziert werden, cf. (das) Blau - der Blauton. Dasselbe gilt für verwandte Quantifizierungstechniken wie bspw. die Kollektivbildung (cf. bspw. Wurzel - Wurzelwerk, Laub-Laubwerk, Ast-Astwerk, Versicherung - Versicherungswesen), deren Technik Assoziation, d. h. Zusammenfassung, Gruppenbildung, genannt wird (cf. Seiler/Lehmann 1982: 43). Sie steht im Gegensatz zur Technik der Dissoziation, die in der Aussonderung eines Elements (sog. Singulativum) aus dem Kollektivum besteht wie bspw. bei Versicherung Versicherungsmann/-mensch, Wache (in der abstrakten Lesart) - Wachmann, Fach - Fachmann, Piste - Pistentiger, Party - Partytier/-mensch/-löwe/-tiger, u. v. a. 
Neben der Vervollständigung des Numerusparadigmas ist auch für die Kategorie Genus ein (wenn auch indirekter, s. u.) Beitrag zur morphosyntaktischen Strukturbildung zu finden, welches erstmals in Heringers (1995) „Leitwortprinzip“ formuliert wurde: So bestimmt das Genus des Hyperonyms in vielen Fällen das Genus des Hyponyms, was insbesondere bei Entlehnungen unter dem Prinzip von Reihenbildung zum Tragen kommt, cf. bspw. der Edding wegen der Stift, das Soda wegen das Wasser, oder der Caipi/Mai-Tai wegen der Cocktail, welches auf der Trank rückführbar ist. In den genannten Fällen liegen - vermutlich aus Gründen lexikalischer Redundanz, so könnte man annehmen, die Köpfe in nicht-realisierter Form vor (der Edding-Stift, das Soda-Wasser, der Caipi-Cocktail). ${ }^{7}$ Die Fälle, in denen die Köpfe realisiert sind (wie bspw. Lindenbaum oder mhd. antvogel, Entenvogel'), werden von Bloomer (1996) als ,pleonastische” Komposita bezeichnet, da aus lexikalischer Sicht keine Notwendigkeit zur Spezifikation besteht (eine Linde ist immer ein Baum, eine Ente ist immer ein Vogel usw.). Aus grammatischer Sicht erweisen sich solche Pleonasmen jedoch als regelbasierte Genusreihenbildungen, die formal über Analogie motiviert sind. Da damit weitere Fälle von Paradigmatizität beim Zweitelement nicht zu finden sind, ist der Blick im Folgenden auf das Erstelement zu richten.

\subsection{Grammatischer Beitrag des Erstelements?}

Um der Frage nach einer grammatischen Strukturbildung der Komposition näher zu kommen, gilt es historisch dokumentierte Diskontinuitäten in Bezug auf einen festgestellten Komplexitätszuwachs hin zu prüfen und diese auf die Grammatikalisierungstheorie abzubilden. Bereits Wilmanns (1896: 515) stellt in diesem Zusammenhang fest, dass komponierte Erstelemente v. a. im Gotischen, aber auch im Ahd., selten waren und dass Komposita erst in Zusammenwirken mit der sog. „uneigentlichen Komposition“ (d. h. ab fnhd. Zeit) im Laufe der Zeit „länger“ (i. S. v. phrasal komplex) wurden. Da beim Erstelement mehr Dynamiken zu beobachten sind (cf. Interfigierung, Dereferenzialisierung, involvierte Wortarten) - wie auch an der Fülle der Literatur synchron wie diachron hierzu abzulesen ist (cf. Kapitel 5) -, ist daraus zu schließen, dass entsprechende Grammatikalisierungsschritte dort zu lokalisieren sind. Im folgenden Kapitel sollen zuerst mögliche synchrone Restriktionen ermittelt und sich daraus ergebende Fragen der morphologischen Analyse präsentiert werden, welche nach der Vorstellung der diachronen Kompositaforschung mit dieser unter dem Aspekt einer möglichen Anwendbarkeit der Grammatikalisierungstheorie hin zusammengeführt werden sollen. Vor diesem Hintergrund soll die Frage erörtert werden, welcher Inhalt zu grammatikalisieren ist.

\section{Gegenwartssprachliche Befunde}

Da bereits für die produktive Suffigierung des Gegenwartsdeutschen genusspezifische Verteilungen von Nominalisierungen anhand der Dimension der (Nicht-)Zählbarkeit identifiziert

\footnotetext{
7 Ähnlich argumentieren auch Köpcke/Zubin (2005), allerdings unter der Annahme von kopflosen NPs. Da aus morphologischer Sicht jedoch der Kopf die kategorialen Eigenschaften des gesamten Kompositums bestimmt im Gegensatz zu Phrasen wie Haus (N.) des Vaters (M.), bei denen herkömmlicherweise sowohl der Kopf als auch das Attribut Referenz erzeugen, erscheint für den vorliegenden Fall die Annahme koverter Komposition plausibler.
} 
werden konnten (cf. Vogel 1996; Leiss 2005; Werner 2012), stellt sich unter mereologischem Blickwinkel unter der Annahme einer Grammatikalisierung die Frage einer grammatischen Semantik auch für die Komposition, was in diesem Fall das Erstelement betrifft, da dieses im Gegensatz zum Zweitelement in nicht-referenzialisierter Form vorliegt und damit den Status eines „wortinternen“ Elements genießt (cf. Abschnitt 3.2). Im Folgenden sollen zunächst formal auf synchroner Ebene gegenwartssprachliche Restriktionen (Abschnitt 4.1) und in einem nächsten Schritt die Wortartendistribution der Komposition (Abschnitt 4.2) thematisiert werden, die beide mit den diachronen Befunden (Kapitel 5) in Kapitel 6 zusammengeführt werden können.

\subsection{Restriktionen als Distributionsbeschränkungen}

Die sich aus Distributionsproben zum Gegenwartsdeutschen ergebenden Restriktionen werden unter morphosyntaktischem Aspekt als formale Restriktionen klassifiziert und seien im Folgenden skizziert. Insbesondere ergeben sich, wie im Folgenden zu zeigen sein wird, Restriktionen bei markierten (d. h. derivierten und flektierten) Konstituenten.

1. Diminution: Diminutiva können nicht als Erstelemente von Komposita fungieren, cf.: *Tischchendecke, aber Tischdecke, ebenso *Bäumchen-Rinde, aber Baumrinde. Ausnahmen dazu sind lediglich Lexikalisierungen wie bspw. Mädchen - Mädcheninternat (cf. Wilmanns 1896: 510).

2. Motion: Movierte Personenbezeichnungen (auf -in) können entweder gar nicht, so im Nhd. (Wilmanns 1896: 514), bzw. aus gegenwartssprachlicher Sicht immer noch selten als Erstelemente fungieren: ? Ärztinkittel, ?Erzieherin-Zimmer. Überwiegend wird in solchen Fällen ein Fugenelement bevorzugt, cf. Ärztin-nen-Kittel, Erzieherin-nen-Zimmer. Diese Entwicklung ist möglicherweise auf den Einfluss der synthetischen Komposition zurückzuführen, da deren Erstelement auf ein internes Argument von $\mathrm{V}^{0}$ verweist und zur Erzeugung generischer Semantik häufig die pluralische Form annimmt wie etwa in Ärztinnen-Streik (> VP Ärztinnen streiken), Erzieherinnen-Lauf ( $>$ VP Erzieherinnen laufen cf. Abschnitt 2.2). Die sich ergebende Verwendungsdifferenz zwischen Wilmanns (1896) obiger Beobachtung und den gegenwartssprachlichen Befunden dürfte m. E. durch den ab der zweiten Hälfte des 20. Jahrhunderts verstärkten Einfluss der feministischen Linguistik und des damit verbundenen frequenteren Gebrauch der movierten Formen befördert worden sein. Insgesamt überwiegen jedoch nicht-movierte Formen deutlich.

3. Nominalisierungen: Die Realisation von deverbalen Nominalisierungen als Erstelemente von Komposita unterliegt teilweise erheblichen Restriktionen und ist auch auf bereits seit Längerem zu beobachtende Produktivitätsdynamiken (so beim Suffix -ung, cf. Demske 2000) bezogen:

3.1 Produktive Derivationsbildungen des Suffixes -erei (wie in Herumchatterei, Mailerei, Knallerei) können nicht als Erstelemente von Komposita fungieren (cf. *Mailerei-Zeit, *Herumchatterei-Wutanfall, *Knallerei-Lautstärke). Stattdessen werden analytische Realisierungen gebildet (wie Zeit für die Mailerei, Wutanfall während der Chatterei, Lautstärke der Knallerei). Eine Ausnahme hierzu stellen Lexikalisierungen dar (wie in Fleischerei- 
FachverkäuferIn, Bäckerei-Eingang, Brauerei-Bilanz), die keinen Restriktionen unterworfen sind (cf. auch Abschnitt 1.2).

3.2 Die Unfähigkeit zur Realisation als Erstelement betrifft auch nicht-pluralisierbare, nominalisierte Infinitive, cf. bspw. *Turnen(s)schuh, *Schwimmen(s)bad, *Entlauben(s)-maschine. Auch hier sind Lexikalisierungen (wie Essen, Leben) jedoch möglich, cf.: Essens-ration, Lebens-baum; die Lexikalisierung ist jeweils an der Pluralfähigkeit der Infinitive zu erkennen.

3.3. Deverbale Nominalisierungen des Suffixes -ung sind gegenwartssprachlich ausschließlich mit dem Fugenelement -s- möglich (wie in Umgehung-s-straße, Abkürzung-s-versuch). ungNominalisierungen als Erstelemente ohne das Fugenelement $-s$ - sind nach eigenen Recherchen im DWDS-Korpus im Gegenwartsdeutschen nicht belegbar, dies gilt auch für -heit/keit/-igkeit-Derivationsbildungen (*Ableitung-versuch, *Schönheit-operation, *Fruchtbarkeitritus, *Kleinigkeit-manöver). ${ }^{8}$ Damit selegieren alle produktiv derivierten Nominalisierungen mit femininem Genus obligatorisch und formal ausschließlich das Fugenelement -s- (cf. auch Abschnitt 2.2) im Gegensatz zu synthetischen Komposita, welche -ung-Nominalisierungen ohne Fugenelement (wie in Stellung-nahme als Derivation zur VP Stellung nehmen) zulassen. Ungeachtet ihrer Interfigierung können -ung-Nominalisierungen als Erstelemente von Determinativkomposita allerdings durch Verbstämme ersetzt werden wie Ableitung-s-silbe $\rightarrow$ Ableit-silbe, Abkürzung-s-versuch $\rightarrow$ Abkürz-versuch, Belegung-s-plan $\rightarrow$ Beleg-plan (cf. auch Kapitel 5). Der Hauptgrund für die zunehmende Präferenz des Stamms dürfte in der zunehmenden Inproduktivität des Suffixes -ung seit fnhd. Zeit liegen (cf. Demske 2000), mit der Folge dass -ung-Nominalisierungen zunehmend Restriktionen auch in Komposita unterliegen (wie bei Anmail-versuch neben ??Anmailung-s-versuch, Schreib-unterlage im Gegensatz zu *Schreibung-s-unterlage, Späh-versuch im Gegensatz zu *Spähung-s-versuch). Das StammKompositionsmuster steht wiederum im Gegensatz zu den usualisierten Komposita mit -ungNominalisierungen (Typ: ?Töt-absicht vs. Tötung-s-absicht). Die Präferenz zur Selektion des Stamms ist bei ad-hoc-Bildungen nach eigenen Stichproben systematisch und somit nicht auf bestimmte Verben beschränkt, cf. Anmiet-vereinbarung, Surf-wettbewerb, Inhalier-spray (neben Inhalationsspray). Zur historischen Dimension cf. Kapitel 5.2 und 5.3. Im Gegensatz zu deverbalen Nominalisierungen können deadjektivische Nominalisierungen als Erstelemente nicht auf ihre Derivationsbasis (cf. Schönheits-OP / *Schön-OP) bzw. den Stamm (cf. Abgeschlagenheit-s-symptomatik / *Abgeschlagen-symptomatik / *Abschlag-symptomatik) reduziert werden. ${ }^{9}$

3.4 Ebenso können nominale Zirkumfigierungen nicht als Erstelemente fungieren: Ort des Herumgehopses, cf. jedoch *Herumgehopse-Ort; ebenso Konsequenz des (dauernden) Ge-

\footnotetext{
${ }^{8}$ Die Herausbildung der sog. ,unparadigmatischen” -s-Fuge ist das Ergebnis eines bereits im Fnhd. stattfindenden Herausbildungsprozesses, dessen Wurzeln nach eigenen Stichproben mithilfe des Austrian Baroque Corpus (cf. https://acdh.oeaw.ac.at/abacus [19.05.2016]) sowie den Ergebnissen von Wanzeck (2003) in paradigmatischen Fugenelementen liegen. Zur Frage der phonologischen Bedeutung cf. Nübling/Szczepaniak (z. B. 2008, 2010).

${ }^{9}$ Aus morphologischer Sicht ist die Frage nach der Reduktion auf die Basis vs. auf den Stamm bei der Komposition von wichtiger Bedeutung. Da es sich bei diesem Reduktionsprozess um einen synchron wie diachron relevanten Parameter handelt, soll die diesbezügliche Diskussion vorerst zurückgestellt und in Abschnitt 6 wieder aufgegriffen werden.
} 
kreisches, cf. jedoch *Gekreische-Konsequenz. Auch hier wird, in Analogie zu den anderen Deverbalia der Verbstamm bevorzugt: Herumhops-Lautstärke, Kreisch-Konsequenz.

3.5 Nomina agentis (wie Lehrer, Schüler, Gärtner) und Nomina instrumenti (wie Bohrer ,x, mit dem man bohrt') sind zwar als Erstelemente von Komposita zu belegen (wie bei Lehrerzimmer, Nitroverdünner-flasche). Wie anhand von Stichproben zu gegenwartssprachlichen Korpora ermittelt wurde, gilt dies jedoch i. d. R. nicht für sog. Nomina acti (Vorgangsbezeichnungen wie Ausrutscher, Hopser, Lacher). ${ }^{10}$ Soll jeweils auf die verbale Situationsungebundenheit Bezug genommen werden, wird, ebenso wie bei allen anderen deverbalen Nominalisierungen (cf. Abschnitt 3.1-3.4), der Verbstamm selegiert, cf. Lach-Lautstärke (im Vergleich zu ??Lacherlautstärke) oder Ausrutsch-Gefahr (im Vergleich zu ??Ausrutschergefahr).

4. Adjektivische Erstelemente: Da von einem Zusammenspiel von sprachinternen und externen Faktoren auszugehen ist (zu Details cf. Schuster 2016: 270-272) ist eine historischen Differenzierbarkeit von $\mathrm{A}+\mathrm{N}-\mathrm{Phrase}$ vs. $\mathrm{A}+\mathrm{N}-\mathrm{Kompositum}$ nicht zuletzt wegen der erst seit dem Fnhd. aufkommenden sprachnormativen Einflussnahme, welche zugunsten der Volletablierung der Adjektivflexion eintrat (Typ: unser täglich Brot $\rightarrow$ unser tägliches Brot, cf. hierzu etwa Pounder 2001), in der Diachronie nicht eindeutig feststellbar. Dies gilt allerdings auch für die Synchronie (Typ: kirchlicher Standesvertreter / Kirchenstandesvertreter, cf. Duden 2009: 348). In jedem Fall zeichnet sich bei der Komposition diachron, aber auch synchron eine stabile Kontinuität zugunsten der Unmarkiertheit des adjektivischen Erstelements ab, was synchron an einigen Restriktionen illustriert werden kann:

4.1 Adjektivderivation: Gemäß Literatur können keine abgeleiteten Adjektive als Erstelemente fungieren, cf. Farbpapier statt *Farbigpapier, Esspapier statt *Essbarpapier, ebenso Kunsthonig statt *Künstlichhonig (cf. Schlücker 2012).

4.2 Komparation: Die formalen Restriktionen betreffen auch die Formen des Komparativ und Superlativ: Diese sind ebenfalls ausgeschlossen (cf. Wilmanns 1896: 514), d. h. hier finden sich keine systematischen Distributionen. Aufgrund der nur punktuellen Belegbarkeit ist zu vermuten, dass einige seltene, hochfrequente Verwendungen synthetischer Komposita (Typ: Gering(st)-verdiener, d. h. synthetische Komposition zur VP gering(st) verdienen) in geringem Umfang die Determinativkomposition mitbeeinflusst haben. Insgesamt sind Belege dieser Art, wie in Stichproben der frequentesten Adjektive des Deutschen anhand des DWDS

\footnotetext{
${ }^{10}$ Die Nomina acti stellen dabei die komplexeste Semantik bereit, da die Semantik von Nomina agentis und Nomina instrumenti daraus ableitbar ist: So kann bspw. ein Hopser neben der usualisierten Bedeutung als Nomen acti auch auf die Bedeutung ,x zum Hopsen' (z. B. ein Kinderspielzeug) sowie ,x, der hopst' kontextuell reduziert werden, umgekehrt jedoch lässt sich ein Nomen agentis wie Lehrer bzw. Nomen instrumenti wie $\mathrm{We}$ cker kontextuell nicht auf die Bedeutung eines Nomen acti bringen wie in ${ }^{*} E r$ hat einen Wecker (,Weckversuch') getan. Daraus ergibt sich eine implikative Hierarchie: Die umfassendste Semantik wird allein von den Nomina acti, nicht aber von den Nomina agentis bzw. instrumenti bereitgestellt. Da diese somit das komplette Spektrum grammatikalisierter Zählbarkeitssemantik abbilden, ergibt sich daraus semantisch wie formal, dass nur diese für die Reduktion bei Setzung in Erstposition repräsentativ sind, denn aufgrund ihrer semantischen Komplexität handelt es sich um die grammatikalisiertesten Nominalisierungen innerhalb des Maskulinums (zu Details cf. auch Abschnitt 6.1).
} 
exemplarisch geprüft wurde, als außerordentlich selten einzustufen. ${ }^{11}$ Formal möglich sind sie allenfalls dort, wo gesteigerte Adjektivformen stets (ggf. implizit) auf ihre nicht gesteigerte Form verweisen: So impliziert bspw. die Form Härtestkäse stets die nicht-gesteigerte Form (wie Hartkäse). In den anhand von Stichproben ermittelten Fällen des DWDS-Korpus (www.dwds.de [19.05.2016]) handelt es sich dabei jedoch stets um usualisierte Bildungen (wie Härtestkäse > Hartkäse, Schwerstgewicht $>$ Schwergewicht, Kleinstwagen $>$ Kleinwagen). Bildungen „ohne Vorlage“, d. h. nicht-usualisierte, d. h. ad-hoc Bildungen (Typ: *Plattestbahn, *Trockenstpapier) konnten anhand von Stichproben nicht ermittelt werden. Dasselbe gilt für Lexikalisierungen (cf. *Tollstwut zu Tollwut, *Bläust(en)beere). In jedem Fall ist der Komparativ auch bei Usualisierungen nicht belegbar, vermutlich da diese Kategorie aufgrund seiner kategorialen Semantik mit der Situationsungebundenheit des Erstelements (cf. Abschnitt 4.2) schwer zu vereinbaren ist. Dies gilt auch für die nächste Gruppe von Belegen im Folgenden.

5. Partizipien: Auch Partizipien können nicht als Erstelement realisiert werden (*Gekochtschinken, *Geklöppelt-spitze), abgesehen von sehr seltenen und insbesondere fachsprachlich motivierten Beispielen wie bspw. Belebtschlamm (neben fachsprachlichem belebter Schlamm); in den sehr wenigen Fällen scheint, wie auch im vorherigen Fall, oftmals die Reduktion einer synthetischen Komposition mit Ausfall der verbalen Konstituente vorzuliegen wie bspw. in Gebrauchtwagen zu Gebrauchtwagenhändler / Gebrauchtwagenhandel zur VP (mit) gebrauchte(n) Wagen handeln; ebenso nach eigenen Recherchen Belebtschlamm zu Belebtschlamm-Verfahren; die Gradation ist hier jedoch ebenfalls nicht möglich (cf. *Gebrauchter-/*Gebrauchtest-wagen, *Gemischter-/*Gemischtest-waren).

6. Adverbien: Da Adjektive und Adverbien mehrheitlich nur syntaktisch unterschieden werden, ist der Status von „echten“ Adverbien (d. h. ohne adjektivisches „Pendant”) als Erstelemente auf den ersten Blick widersprüchlich. Allerdings lassen sich einige Bildungen finden, die für begrenzte Produktivität sprechen (wie Jetzt-Zeit, Kaum-Bluse), obgleich zu hochfrequenten Adverbien analytische Alternativkonstruktionen zu existieren scheinen, cf.: *HeuteTag, aber heutiger Tag, ?Vorher-Termin, aber vorheriger Termin oder ?Hier-Raum, aber hiesiger Raum. Dies zeigt, dass die Reihenbildung der Adv+N-Komposition zwar produktiv, allerdings aufgrund der niedrigen Zahl von Adverbien ohne adjektivisches „Pendant” als niedrigfrequent einzustufen ist. Einige der nach Korporaauswertungen in Tokens hochfrequenten, aber in Types niedrig frequenten Belege wären u. U. auch über Kontaminationseffekte der synthetischen Komposition (wie der Hier-Steher, Jetzt-Esser, Schnell-Trinker) bzw. Konversion motivierbar. Markierte Satzadverbien können hingegen nicht als Erstelemente

\footnotetext{
${ }^{11}$ So finden sich bspw. im Deutschen Textarchiv (DTA, zugänglich über www.dwds.de [19.05.2016]) bei der Konstituente Kleinst- ab dem 17. Jahrhundert kein Type, der strukturell nicht auf eine synthetische Komposition rückführbar wäre. Im dort verlinkten ZEIT-Korpus des $20 \mathrm{Jh}$. finden sich hierzu hingegen mehr Belege (knapp 2000 Token, allerdings inklusive der mehrheitlich synthetische Komposita mit anderen distributionellen Eigenschaften, cf. Abschnitt 2.2). Im Gegenwartsdeutschen muss die Produktivität von komparierten Adjektivformen für die Determinativkomposition somit bezweifelt werden. Wilmanns (1896: 514 f.) bemerkt hierzu: „Aber erst im Nhd. treten diese Wörter [die Wörter mehr und minder] von neuem hervor und neben ihnen andere: Mehrzahl, Minderzahl, Minderbrüder, mehrjährig, mehrdeutig, minderjährig. Loser sind die Verbindungen adjectivischer Participia mit adverbial gebrauchten Superlativen wie meistbietend, minderberechtigt u. ä.“ [Hinzufügung M.W.] Hier lässt sich also für die Gegenwartssprache, nicht aber diachron (s. o., cf. Wilmanns 1896: 514) eine Beeinflussung durch die synthetische Komposition nicht völlig ausschließen.
} 
fungieren cf. *Glücklicherweise-Sonne, *Merkwürdigerweise-Pflanze, *VerwunderterweiseKatze. Dies gilt auch für mit -(ling)s suffigierte Adverbien (wie nachts, blindlings, tags), cf. *Nachts-lampe, *Tags-Zigarette oder *Blindlings-Kellner.

Auch der letzte Befund fügt sich in die Grundregel, dass produktiv derivierte bzw. flektierte Wörter mit starker Tendenz nicht als Erstelemente fungieren können oder, wie im Fall von heit/(-ig)keit und -ung, aber auch der teillexikalisierten Suffixe -schaft und -tum (wie Studenten-schaft/-tum-s-verhältnis), mit -s- interfigiert werden. Auf Basis der genannten, formalen Restriktionen der Determinativkomposition lassen sich erste Rückschlüsse im Hinblick auf die Semantik des Erstelements ableiten, die im nächsten Abschnitt eingehender betrachtet werden sollen.

\subsection{Synchrone Wortartenbeteiligung}

Gemäß Literatur wurde festgestellt, dass Komposita des Deutschen über Generizität beschrieben werden können, insbesondere, da die Erstelemente von Komposita dereferenzialisiert vorliegen (cf. etwa Demske 2001; cf. auch Abschnitt 5.1 und 5.2 zur Diachronie): So referiert bspw. das Erstelement des Kompositums Vogelnest nicht auf einen bestimmten Vogel oder bestimmte Vögel, sondern es liegt dereferenzialisiert vor und bezieht sich auf den „Vogel an sich" (auch sog. klassifikatorische, d. h. typisierende Komposition nach Schlücker 2014). Die im Erstelement zugrundeliegende generische Semantik ist dabei auch bei allen anderen, nichtnominalen Wortarten festzustellen und steht in Opposition zu einer analytischen Realisation mit entsprechender nicht-generischer (episodischer bzw. referenzieller) Semantik, cf. Tabelle 1.

\begin{tabular}{|l|l|l|}
\hline & Komposition & Analytische Struktur \\
\hline $\mathbf{N}$ & Hutschachtel, Hundehalsband & $\begin{array}{l}\text { Schachtel für den/einen Hut, Halsband } \\
\text { für den/einen Hund }\end{array}$ \\
\hline Eigennamen & $\begin{array}{l}\text { Mozart-Stück, Dürer- } \\
\text { Riesenpuzzle }\end{array}$ & $\begin{array}{l}\text { Stück von Mozart, Riesenpuzzle nach } \\
\text { Dürer }\end{array}$ \\
\hline $\mathbf{A}$ & Starkregen, Kleinwagen & starker Regen, kleiner Wagen \\
\hline $\mathbf{A d v}$ & $\begin{array}{l}\text { Zuhause-Mantel, Zuvor- } \\
\text { Zigarette }\end{array}$ & Mantel zuhause, Zigarette zuvor \\
\hline $\mathbf{V}$ & Schwimmkerze, Inhalierspray & $\begin{array}{l}\text { schwimmende Kerze, zu inhalierendes / } \\
\text { inhaliertes Spray }\end{array}$ \\
\hline Interjektion & Igitt-Tier, Pfui-Tag & $\begin{array}{l}\text { Tier/Tag, das/der mit der Äußerung } \\
\text {,igitt“/“pfui“ in Verbindung steht }\end{array}$ \\
\hline $\mathbf{X ( P )}$ & $\begin{array}{l}\text { Alles-oder-nichts-Regel, (der) } \\
\text { lass-uns-Freunde-bleiben-Satz }\end{array}$ & $\begin{array}{l}\text { (die) Regel ,,alles-oder-nichts “, } \\
\text { (der) Satz ,,lass uns Freunde bleiben “ }\end{array}$ \\
\hline
\end{tabular}

Tabelle 1: Komposita (nach Wortart des Erstelements sortiert) in Opposition zur analytischen Realisation

Eine Bildung wie Schwimmkerze bezeichnet damit eine ,Kerze, die (per se) schwimmen kann”. Die Bildung Starkregen bezieht sich nicht nur auf eine bestimmte ,Art von Regen”, denn diese Bedeutung würde auch durch das Attribut stark hergestellt werden, sondern sie bezeichnet eine bestimmte Klasse generischer Qualität (,Regen, der per se stark ist"). Wie darüber hinaus in Tabelle $1 \mathrm{zu}$ erkennen ist, sind die letzten beiden Kategorien (Interjektion 
und $\mathrm{X}(\mathrm{P}))$ in ihrer analytischen Realisierung entsprechend graphematisch markiert wiedergegeben. Dies erfolgt in Übereinstimmung mit der Interpretation von Wiese (1996), demzufolge diese als metalinguistische Einheiten (im Sinne von Zitaten) zu interpretieren sind. Besonders auffällig wird dies bei der Kategorie der Interjektion, deren Ansetzen eines entsprechenden analytischen Korrelats ausschließlich mit einer die metasprachlichen Anteile berücksichtigende Paraphrase sinnvoll wiederzugeben ist, da ansonsten morphosyntaktische Restriktionen vorliegen (cf. *igittes Tier / *Tier des ,,igitt“ / *igittendes Tier / ?Tier mit ,,igitt“). Wie an diesem Beispiel zu ersehen ist, erfordern Komposita mit metasprachlichen Einheiten Vorwissen, welches vom Sprecher als bekannt vorausgesetzt wird bzw. bei der Dekodierung vom Hörer auf Basis seines Weltwissens (hinsichtlich konventionalisierter Sätze oder Idiome) inferiert werden muss.

Aus semantischer Sicht ist zudem auf den ersten Blick die Integration von Eigennamen in Komposita auffällig, was gemäß Zifonun (2010) auf den Einfluss des Englischen zurückzuführen ist. Bei den entsprechenden Bildungen ist nicht nur eine possessive Interpretation, sondern auch eine Art-Interpretation möglich (wie bei Dürer-Riesenpuzzle, bei der die Interpretation ,Puzzle von Dürer', zwar formal möglich, jedoch u. a. aufgrund von Inferenz durch Weltwissen ausgeschlossen werden kann, cf. Tabelle 1). Viele Komposita sind im Vergleich $\mathrm{zu}$ ihren analytischen Bildungen jedoch einem usualisierten Gebrauch zuzuordnen, so z. B. bei Schnellrestaurant vs. schnelles Restaurant, ebenso wie Blaubeere vs. blaue Beere, Dunkelkammer vs. dunkle Kammer etc. Kontextfreie ad-hoc-Bildungen ohne Markierungen, z. B. auf Lexikalisierung (cf. Nübling/Szczepaniak 2008: 3) hindeutende Fugenelemente, sind inhärent polysem, d. h. erst durch den jeweiligen Aktualisierungskontext eingeschränkt, so etwa bei Einstein-Idee mit der Bedeutung ,Idee von Einstein' sowie ,Idee in der Art von Einstein', ,einsteinische Idee'.

Vor dem Hintergrund dieser Beobachtung ergibt sich, dass bspw. ein Kompositum wie Kreuzallergie aufgrund des Erstelementes, welches im Gegenwartsdeutschen formal unterschiedlichsten Wortarten zugeordnet werden kann, ohne einen spezifischen Aktualisierungskontext oder Usualisierung unterschiedlichsten Interpretationen zugeordnet werden kann, so bspw.: ,Allergie auf ein Kreuz/auf Kreuze', ,Allergie, die etwas kreuzt/bei der sich etwas kreuzt', ,zu kreuzende Allergie', ,Allergie von Herrn/Frau Kreuz', ,Allergie nach Art eines Kreuzes/mit Kreuzen (Kreuzmustern, o. Ä.)'. Im Gegensatz zu Lexikalisierungen, bei welchen lediglich eine einzige Interpretation verfügbar ist (wie bei Erdbeere *,Beere der Erde') kann in diesem Fall aufgrund von Usualisierung die festgelegte Interpretation ausgewählt werden (in diesem Fall ,Allergie, die etwas kreuzt/bei der sich etwas kreuzt'), jedoch kann ebenso gut eine durch einen anderen Kontext nahegelegte Interpretation möglich sein. Ein Beispiel eines ad-hoc-Hörbelegs, welches ohne Kontext frei interpretierbar ist, wäre die Bildung Fuchtelforscher (mündlicher Beleg) mit den möglichen Paraphrasen ,Forscher, der fuchtelt", ,Forscher, der sich mit dem Fuchteln (d. h. Gestik) beschäftigt', ,Forscher, der unter jmds. Fuchtel steht (idiomatisiert)'. Vor dem Hintergrund der formalen Distributionen stellt sich im Folgenden die Frage ihrer möglichen Entwicklung. 


\section{$5 \quad$ Blick in die Diachronie}

Die Annahme einer Grammatikalisierung der Komposition stößt bereits auf die Schwierigkeit ihrer Datierung (zu Details cf. Pavlov 2009; Schlücker 2012): Während von einigen Forschern das (Vor-)Ahd. als Sprachstufe mit Produktivwerdung der Komposition angesetzt wird (cf. bspw. Osthoff 1878; Carr 1939; auch sog. „eigentliche Komposition“ in Grimm'scher Terminologie), wird von anderer Seite das Fnhd. als Stufe der Produktivwerdung angenommen (so bspw. Demske 2001; Wanzeck 2003; auch sog. „uneigentliche Komposition“ bei Grimm). Als Argument für die spätere Datierung wird v. a. die im Fnhd. zu beobachtende Desyntaktisierung bzw. Dereferenzialisierung eines pränominalen Genitivattributs (wie heute noch in des Rätsels Lösung) angeführt, welches bei der Entstehung der Komposition als Erstelement eines Kompositum reanalysiert wird. Durch diese formale und insbesondere im Fnhd. zu beobachtende Entwicklung ist die Unterscheidung zwischen Nominalsyntagma und Determinativkompositum nicht immer eindeutig feststellbar, wie bspw. fnhd. des schue rymen , die Riemen seines Schuhs', mit der wibis milche, den waren gotes sun (aus Prell 2000: 28f.). Die Frage, inwieweit Belege (wie senefes korn, tages lioht, wolfes miluh, aus Demske 2001: 298) aus früheren Sprachstufen als phrasale Strukturen oder aber als Komposita zu interpretieren sind, resultiert zum Einen aus der fehlenden orthographischen Normierung (cf. Solling 2012; cf. in diesem Zusammenhang bspw. auch die bis heute bestehende Getrenntschreibung von Komposita im Englischen wie in book shelf, vacuum cleaner, doll factory), zum Anderen aber auch daraus, dass die exakte Semantik des pränominalen Genitivs trotz vieler Versuche bislang nicht geklärt werden konnte und daher für weitere Diskussion vorerst auf die Literatur verwiesen werden muss (zu einem Überblick cf. Prell 2000). In den folgenden Abschnitten jedoch sollen unter Berücksichtigung bislang vernachlässigter, formaler Aspekte unter der Zusammenführung mit bereits bekannten Eigenschaften neue Schlüsse gezogen werden, die die Annahme einer Grammatikalisierung nachzeichnen und parallel dazu u. U. auch die Frage der Datierung der Komposition unter einem anderen Licht erscheinen lassen.

\subsection{Genitivattribut oder Erstelement?}

Die Annahme einer Produktivwerdung der Komposition in fnhd. Zeit ist mit der seit ahd. Zeit andauernden Grammatikalisierung des Artikelsystems verschränkt. Da es jedoch auch Sprachen mit Komposition, aber ohne Artikelsystem (wie bspw. das Vietnamesische) sowie Sprachen mit Artikelsystem, aber ohne Komposition (wie bspw. das Ungarische ${ }^{12}$ ) gibt, zeigt, dass beide Phänomene formal unabhängig sind, $d$. h. automatisch nicht einander grammatisch implizieren. Im folgenden Abschnitt soll jedoch basierend auf dieser Überlegung der Versuch unternommen werden, Argumente für eine frühere Datierung der Komposition auf Basis bis-

\footnotetext{
12 Auch wenn einige Komposita des Deutschen in das Ungarische entweder direkt entlehnt oder lehnübersetzt werden, ist hier von allenfalls eingeschränkter Produktivität auszugehen, da Komposita des Deutschen im Ungarischen überwiegend mit Syntagmen wiedergegeben werden, cf. bspw. dt. Nussschokolade - ung. mogyorós csokoládé wörtl. ,nussige Schokolade'; dt. Käsekuchen - ung. kúrós sütemeny wörtl. ,käsiger Kuchen'; dt. Weihnachtsdekoration - ung. karácsonyi dekoráció wörtl. ,weihnachtliche Dekoration'. Noch deutlicher wird die morphologische Differenz zwischen beiden Sprachen bei dt. V+N-Komposita, deren Erstelement im Ungarischen dem Partizip Präsens entspricht wie ír-ó-asztal wörtl. ,schreibender Tisch', dt. Schreibtisch; olvas-ó-könyv wörtl. ,lesendes Buch', dt. Lesebuch sowie lát-ó-ideg wörtl. ,sehender Nerv', dt. Sehnerv.
}

ISSN 1615-3014 
heriger Beobachtungen der Literatur sowie eigener Stichproben (zur Methodologie cf. Abschnitt 1.2) zu entwickeln.

In Bezug auf die Dereferenzialisierung des Erstelements im Fnhd. hat bislang Demske (2001) aus syntaktischer Perspektive die umfassendste Arbeit zum Stellungswechsel innerhalb der Nominalphrase, mit Berücksichtigung der Synthetisierung hin zur Komposition, vorgelegt. Ihr zufolge besteht die Funktion der Komposition in der Bereitstellung von generischer Semantik des Erstelements im Gegensatz zu analytischer Realisierung wie bspw. Fensterrahmen ,Rahmen von Fenstern' vs. Rahmen des/dieses Fensters, cf. hierzu genauer Abschnitt 4.2. Der im Fnhd. zu beobachtende Prozess der Synthetisierung aus analytischen Strukturen (pränominales Genitivattribut) zur Bildung der Komposition impliziert vor dem Hintergrund der in Abschnitt 4.2 herausgearbeiteten Semantik von Generizität, dass das Artikelsystem zu dieser Zeit schon soweit grammatikalisiert war, dass der definite Artikel, dessen Grundfunktion mehrheitlich in der Kodierung von Bekanntheit (cf. Leiss 1992: 121; Heusinger 2010) besteht, bereits auch in generischen Kontexten verwendet werden konnte. Das bedeutet jedoch auch umgekehrt, dass in früheren Sprachstufen nominale Generizität auch ohne Artikel realisiert wurde, da dieser noch gar nicht in dieser Funktion verwendet werden konnte. Daher können auch pränominale Genitivattribute ohne Artikel generische Semantik zum Ausdruck bringen, so dass eine frühere Datierbarkeit der Komposition auf das Ahd. möglich wird. ${ }^{13}$ Der einzige, formale Unterschied zwischen beiden Mustern besteht demnach darin, dass „uneigentliche“ Komposita Fugenelemente besitzen, die auf ehemalige Kasusflexive zurückgeführt werden können, während frühere, „eigentliche“ Komposita sog. Stammkomposita sind (cf. Solling 2012: 35).

Die Tatsache jedoch, dass sich über das Merkmal der Generizität nur indirekt Aussagen in Bezug auf einen möglichen Einfluss der Definitheit in fnhd. Zeit machen lassen, dass gleichzeitig artikellose pränominale wie postnominale Genitive ebenso wie die Komposition nominale Generizität enkodieren können, kann im Lichte der Grammatikalisierungstheorie als sog. „Layering“ interpretiert werden: Alte Formen (Nominalsyntagma) und neue Formen (Komposition) überlagern sich ohne Bedeutungsdifferenzierung. Damit ist es aus Grammatikalisierungssicht unerheblich, ob die jeweiligen Formen analytisch (wie im Syntagma) oder synthetisch (wie in der Komposition) vorliegen, da die Semantik nominaler Generizität gewährleistet ist. Auch aufgrund der Tatsache, dass ahd. Belege in nicht-referenzieller und damit generischer Verwendung dokumentiert sind (wie ahd. tagalioht, Tageslicht', aber eben nicht ,Licht eines bestimmten Tages', berg-fugeli ,Bergvogel', eben nicht, Vogel eines bestimmten Berges' usw.), spricht dafür, dass erste Vorkommen der Komposition im Ahd. festzumachen sind. Es lassen sich zudem jedoch noch weitere Argumente aus der Morphologie gewinnen.

Da noch bis in Mhd. die Wortarten Substantiv und Adjektiv weniger morphologisch als syntaktisch voneinander geschieden waren (cf. z. B. auch heute noch scheu vs. Scheu), kann die parallele Existenz von $\mathrm{A}+\mathrm{N}$ - und $\mathrm{N}+\mathrm{N}-$ Komposita im Ahd. motiviert werden. Da im Gegensatz dazu keine V+N-Komposita im Ahd. (cf. Carr 1939), jedoch hochfrequent im Gegenwartsdeutschen (Typ: Inhalierspray, cf. Abschnitt 4.2) dokumentiert sind, ist von einem

\footnotetext{
${ }^{13}$ Das bedeutet nicht, dass postnominale Genitive im Deutschen keine generische Bedeutung ausdrücken konnten, doch diese sind aus syntaktischen Gründen strukturell nicht zum Aufbau zur Komposition höherer Komplexität prädestiniert (cf. Abschnitt 1.2 und 2.2).
} 
Sprachwandel auszugehen, der im folgenden Abschnitt expliziert werden soll. Unter dem Aspekt von morphosyntaktischem „Layering“ stellt sich für diese Sprachstufe die Frage, in Form welcher morphologischen Realisation verbale Bestandteile mit generischer Semantik in der Komposition Platz fanden und ob sich - in Analogie zu Layering der genitivischen Formen (s. o.) - Vergleichbares finden lässt. Um diese Frage beantworten zu können, ist es jedoch zuerst notwendig, weitere Aspekte zur Wortartendiachronie der Komposition zu thematisieren. Diese Frage soll daher vorerst zurückgestellt und am Ende von Abschnitt 6.3 wieder aufgegriffen werden.

\subsection{Wortartendiachronie des Erstelements}

Historisch für das Deutsche wurde festgestellt (cf. Behaghel 1923/1932: 177), dass pränominal nur zählbare Nomina in attributiver Funktion in Form des Genitivus possessivus auftreten konnten, nie jedoch nicht-zählbare (sog. Massen- bzw. Stoffnomina). Letztere wurden postnominal realisiert in Form des Genitivus partitivus (wie mhd. ein becher wînes). ${ }^{14}$ Damit wären für die Entstehung der Komposition ausschließlich zählbare, nicht jedoch nicht-zählbare Nomina von Bedeutung. Morphosyntaktisch auffällig in Bezug auf die Frage Komposition vs. Nominalsyntagma ist in diesem Zusammenhang jedoch, dass sich bereits in ahd. Zeit zahlreiche Belege mit Massennomen finden lassen, deren pränominale Realisierung im Sinne einer phrasalen Struktur damit und auch aufgrund der generischen Interpretation ausgeschlossen werden muss. Cf. ahd. Komposita wie miluh-kubil ,Milcheimer, Eimer für Milch', wazzar-faz ,Wassergefäß' (cf. Splett 1993 unter den jeweiligen Schlagwörtern). Da sich im Ahd. auch nach eigenen Stichproben neben Partikel-N-Komposita, die seit dieser Zeit unverändert bestehen und damit keine Charakteristika von Sprachwandel aufweisen (wie nhd. Vorraum oder Nebenschauplatz), ausschließlich $\mathrm{A}+\mathrm{N}$ sowie $\mathrm{N}+\mathrm{N}-\mathrm{Komposita}$ finden lassen (cf. hierzu Carr 1939), kann hierbei von einer eingeschränkten, aber dennoch feststellbaren Produktivität des Wortbildungsmusters Komposition gesprochen werden (Muster: $\mathrm{N}+\mathrm{N}$ wie ahd. äband-zìt ,Abendzeit', zagal-bein ,Schwanzknochen'; A+N edīl-man wörtl. ,Adel-/Edel-mann', ,Mann

\footnotetext{
${ }^{14}$ Im Gegensatz dazu weist Prell (2000: 30) auf die Ergebnisse einer (bis dato) nicht publizierten Untersuchung von Westvik (dort zitiert) hin, der zufolge Partitive im Altgermanischen auch pränominal vorkommen konnten. In diese Richtung argumentiert auch Demske (2001: 231). Nach eigenen Stichproben im Bonner Frühneuhochdeutsch Korpus (cf. www.korpora.org [19.05.2016]) lassen sich im Fnhd. keine entsprechenden Beispiele finden, cf. etwa (Thüringisch, 2. Hälfte des 15. Jh., Johannes Rothe, Chronik, 19/7): „[...] her [Noah] vant eynen weynstuck, der fruchte her vor nicht mer gesehn hatte, unde smackte die weinber das sie gut unde susse waren. Do druckete her sie uss unde machte most unde trangk sin, das her truncken wart, wenn her des weynes craft nicht kante, $[\ldots]$. .,$[\ldots]$ er [Noah] fand einen Weinstock, dessen Früchte er zuvor nie gesehen hatte, und probierte die Weinbeeren [und befand], dass sie gut und süß waren. Da presste er sie aus und machte Most und trank ihn, so dass er betrunken wurde, da er die Kraft von Wein nicht kannte, [...]" [Hervorhebung M.W.]. Die Stelle selbst ist eine indirekte Wiedergabe des 1. Buch Mose, Kap. 9, 20f. und wäre deutlicher wiederzugeben mit ,da ihm die Kraft von Wein unbekannt war", was die generische Qualität der hier vorliegenden Genitivkonstruktion unterstreichen würde, die jedoch trotz der Beteiligung des Stoffnomens Wein nicht partitivisch ist. Für die Generizitätsinterpretation spricht auch die hier vorliegende Verwendung des Präteritums als Erzähltempus, während die Verwendung von Perfekt die Episodizität des Geschehens (und damit Einmaligkeit im Sinne von ,x ihm unbekannt in diesem Fall”) konzeptualisieren würde. Weitere Fälle pränominaler, ,echt" partitivischer Genitivkonstruktionen gelten damit als nicht belegt und stellen damit weiterhin ein empirisches Forschungsdesiderat dar.
} 
von hohem Rang', tarn-winkil ,Schlupfwinkel, Versteck', cf. Splett 1993, unter den jeweiligen Stichwörtern).

Im Mhd. ist dieses Szenario größtenteils unverändert, obgleich sich hier bereits einige $\mathrm{V}+\mathrm{N}$ Komposita finden lassen, deren Erstelemente nicht wie im Ahd. auf ein deverbales Nomen

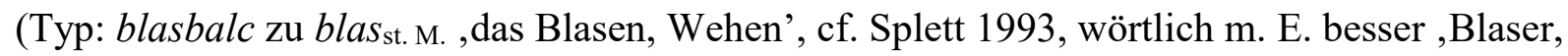
Luftstoß') rückführbar sind. Die entsprechenden, bei Komposita des Mhd. erstmals erscheinenden Erstelemente ohne ein nominales Korrelat können somit als „echte“ verbale Konstituenten gewertet werden. Die Ergebnisse passen zu der Beobachtung von Osthoff (1878), bestätigt durch Wilmanns (1896: 518) und Carr (1939), wonach in allen germanischen Sprachen zuerst $\mathrm{N}+\mathrm{N}-$ Komposita und - soweit aufgrund fehlender Grammatikalisierung von Substantiven unterscheidbar - A+N-Komposita und erst in der Folge V+N-Komposita belegt werden können. Osthoff (1878) zufolge geschah dies, indem deverbale Nullderivationen (wie ahd. blas, st. M.) als Erstelemente verwendet wurden, und durch ihre morphologische Ambiguität (deverbales Nomen vs. Verbstamm) als Verbstämme interpretiert werden konnten (cf. auch z. B. Schlafzimmer ,Zimmer für den Schlaf' und ,Zimmer zum Schlafen', Knallerbse ,Erbse für einen Knall' und ,Erbse, die knallt'). In der weiteren Folge konnten, so lässt sich folgern, diesem Entwicklungsschritt auch $\mathrm{V}^{0}$ (wie das Teller-leer-ess-Kind) und in der Folge sogar unterschiedlichste Phrasen, auch im Sinne metasprachlicher Einheiten i. S. v. X ${ }^{0}$ (wie das ich-will-nicht-krank-werden-Buch) in das Kompositionsmuster integriert werden.

Strukturell umfassen die für den Komplexitätsaufbau relevanten Schritte somit zwei Aspekte: a) die Reinterpretation (in der Grammatikalisierungstheorie auch als Reanalyse bezeichnet) von deverbalen Substantiven als Verbstämme (wie schlaf-, knall-) einschließlich einer damit einhergehenden, kontinuierlichen Erweiterung der Wortarten der Erstelemente (im Sinne einer Wortarten-Neutralisation) sowie b) die Beseitigung von Restriktionen in Bezug auf produktiv nullderivierte, maskuline Nominalisierungen. Nach eigener Sichtung der Belege in Lexer (2011) kann man m. E. daneben noch eine weitere Erklärungsmöglichkeit für die Wortartenerweiterung von $\mathrm{N}$ auf V (oder kurz: $\mathrm{N} \rightarrow \mathrm{V}$ ) geltend machen, die beide Aspekte (a und b) verbindet und die im Folgenden ausführlich dargelegt werden soll.

\subsection{Abstraktnominalisierungen als Drehscheibe für morphologischen Wandel}

Unter der Annahme, dass maskuline Nullsuffigierungen vom Typ Knall, Schlaf in das Kompositionsmuster integriert und als $\mathrm{V}+\mathrm{N}-\mathrm{Komposita}$ reinterpretiert wurden, bleibt offen, warum nur Maskulina die Wortartenerweiterung der Kompositionserstelemente befördert haben sollen, insbesondere vor dem Hintergrund des reichhaltigen suffixalen Inventars der neutralen und femininen Abstraktnominalisierungen. Wie in diesem Abschnitt argumentiert werden soll, liegt der Schlüssel zur Lösung dieses morphologischen Rätsels hinsichtlich der Ausweitung der morphologischen Produktivität nicht nur in der Reinterpretation von Nullsuffigierungen, sondern auch bei den explizit derivierten Nomina, insbesondere den Feminina und Neutra. ${ }^{15}$ Da dieser Aspekt bislang mehrheitlich übersehen wurde, verdient er im Folgenden besondere Beachtung.

\footnotetext{
${ }^{15}$ Eine ähnliche Interpretation im Hinblick auf den Versuch einer Anbindbarkeit entsprechender Konstituenten an deverbale Nomina nimmt die Untersuchung von Gröger (1911, s. z. B. § 27) zu den Fugenvokalen vor.
}

ISSN 1615-3014 
Ursprünglich konnten produktiv derivierte Wörter nicht als Erstelemente fungieren, was zumindest in Bezug auf die Nomina völlig komplementär zum Gegenwartsdeutschen ist: So war in früheren Sprachstufen, insbesondere im Ahd., die Integration dieser jüngsten Schicht derivierter Wörter nicht möglich, was auch jedoch nur noch für Adjektive (cf. Kapitel 6), nicht aber für die hoch grammatikalisierten suffigierten Substantive (Typ: Umgehung-s-straße, Schönheit-s-fleck) zutrifft (cf. Wilmanns 1896: 514):

Im ganzen wird man sagen können, dass abgeleitete Wörter um so weniger zu ersten Compositionsgliedern taugen, je lebendiger die Ableitungssilben als Mittel der Wortbildung empfunden werden, und dass zweite Compositionsglieder, die selbst zu Ableitungssilben herabsinken, wie lich, -tum, -heit gleichgültiger gegen die Form der ersten Compositionsglieder sind als andere. So finden wir im Ahd. zwar Substantiva wie wazzar, fingar, adal, himil, dëgan, morgan, widamo, magad, mânôd, houbit, obez, âbant, jugund, thionost, herbist, phenning, kuning u. ä. als erste Compositionsglieder, aber Substantiva mit lebendigen Suffixen wie -âri, -ida, -unga, -nissi [nhd. -er, -de, -ung, -nis] werden an dieser Stelle gemieden.

[Hinzufügung M. W.]

Da produktiv derivierte Substantive im Gegenwartsdeutschen als Erstelemente fungieren können, historisch jedoch nicht, liegt die Annahme eines Sprachwandels nahe. Damit kommt der Integration derivierter Nomina in das Muster der Komposition diachron wie synchron ein entscheidendes Gewicht in Bezug die Grammatikalisierung zu. Denn produktiv derivierte Abstrakta sind auch historisch grammatisch motivierte, nominale Propositionen, d. h. sie besitzen die Eigenschaft zu prädizieren. Daher sind sie reduzierbar auf Argumentstellen im Satz. Nur bei synthetischen Komposita können sie die Rolle des internen Arguments einnehmen (cf. Abschnitt 2.2). Aufgrund ihrer formalen wie semantischen Komplexität im Sinne grammatikalisierter Abstraktheit (cf. auch Abschnitt 3.2) ist die Integration von grammatisch markierten Abstrakta in das Muster der Determinativkomposition somit ein zentraler Punkt für die semantische Struktur, da dadurch deverbale Konstituenten ihrerseits Argumentstellen deverbaler Derivationsbasen bilden können wie in (diese ewige) Betreuungen-überprüfung (> VP Betreuungen überprüfen), so dass damit eine diachrone Zunahme der Länge von Komposita motiviert und über eine Kreuzung zwischen Determinativkomposition und synthetischer Komposition erklärt werden kann.

Nicht-derivierte Bildungen konnten im Ahd. obigem Zitat zufolge, auch wenn diese im Gegenwartsdeutschen mit einem Suffix wiederzugeben wären (wie Finsterkeit, Finsternis) als Erstelemente realisiert werden (wie ahd. finstar-land, Land der Finsternis' zu ahd.

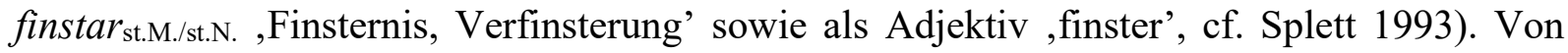
der im Zitat angesprochenen Positionierungsrestriktion der derivierten Nomina nicht betroffen sind jedoch die früher derivierten, deverbalen Abstrakta auf ahd. $-\bar{a}$ (wie in pfressā-baum wörtl. ,Pressungs-baum', ,Dreharm der Kelterer' zu ahd. $p(f)$ ressa $\bar{a}$,Pressung', cf. jeweils Splett 1993) bzw. $-\bar{\imath}$ (wie in redī-spah̄̄, Redegewandteheit, Beredtsamkeit" und daneben als Adj.; zu red̄̄ ,Auseinandersetzung, Streit, Glaubensgrundsatz'; cf. Splett 1993). Entsprechende Komposita mit so suffigiertem Erstelement lassen sich zwar häufiger im Mhd. (lautlich nun als $-e$ ) finden. Der Befund ist insofern interessant, als dass sich im Mhd. erstmals ein Nebeneinander von Belegen mit und ohne - $e$ - beobachten lässt, ein Umstand, der bisher rein auf phonologischer Ebene diskutiert wurde (cf. z. B. Wilmanns 1893: 289f.), nun aber morphologisch interpretiert werden kann; ein Beispiel für dieses Nebeneinander wäre etwa mhd. slahtehus neben slaht-hus, gemäß nhd. Übersetzung zueinander synonym, mit der Bedeutung

ISSN $1615-3014$ 
,Schlachthaus" oder ebenso schabe-mezzer neben schab-mezzer ,Schabmesser," ${ }^{16}$. Die alternierenden Formen können vor dem Hintergrund möglicher (Nicht-)Restriktionen von Suffigierungen als morpho(no)logischer Reduktionsprozess des Formans -e-interpretiert werden, durch welchen nicht nur die kategoriale Information $[+\mathrm{N}]$ verloren geht, sondern gleichzeitig auch die morphologisch neue Interpretation [+V] möglich wird. Parallel dazu wird die einstige Derivationsbasis als Stamm reinterpretiert. Da in der Folge auch $\mathrm{V}^{0}$ und in der Folge $\mathrm{X}^{0}$ als Erstelemente erscheinen, liegt damit ein gleichzeitiger (cf. Abschnitt 4.2) Neutralisationsprozess mit Konsequenzen für die kategoriale Information und Quantifizierung des durch die Reduktion „übrigbleibenden“, lexikalischen Elements vor (cf. auch Abschnitt 6.2). Damit ist auch der Blick diachron nicht nur auf das Belegbare, sondern gleichermaßen auf das Reduzierte bzw. nicht (mehr) Belegbare ${ }^{17}$ gerichtet. Gleichzeitig lassen sich aus gegenwartssprachlicher Perspektive hierdurch vielleicht auch die zahlreichen Belege mit deverbalem Erstelement und „arbiträrer“ risch im Sinne einer Lexikalisierung eines einst überaus motivierten und frequenten Musters erklären, bei dem sich alte und neue Formen wortintern, d. h. innerhalb der Komposition, überlagen (sog. „Layering“ in der Grammatikalisierungstheorie) und nullsuffigierte mit suffigierten Erstelementen um Herstellung nominaler Generizität konkurrierten.

Auch aus syntaktischer Sicht gibt es in diesem Zusammenhang übereinzelsprachliche Evidenz dafür, dass den deverbalen Abstraktnominalisierungen (im Deutschen v. a. mit femininem

16 Um den Beleg möglichst wortgetreu ins Gegenwartsdeutsche zu übersetzen, müsste man eine deverbale Nominalisierung mit femininem Genus wählen, da lediglich feminine Abstrakta im Gegenwartsdeutschen nicht reduziert werden und ein Fugen-s- zu sich nehmen (zu Details cf. Abschnitt 2.2). Die mhd. Bildungen wären somit am ehesten mit ,Schlachtungshaus' bzw. ,Schabungsmesser' wiederzugeben. Dass zahlreiche Konkreta des Gegenwartsdeutschen auf einstige deverbale Nominalisierungen zurückgehen (wie Fliege $\rightarrow$,Fliegung”, usw.) ist hinreichend beschrieben (cf. Prinzip abstractum pro concreto, cf. z. B. Werner 2010) und spricht für die morphologische Bedeutung dieses Musters für den Ausbau der Produktivität und Komplexitätserhöhung der Komposition.

17 Härtl (2015) zufolge lassen sich auch im Gegenwartsdeutschen Alternierungen von V+N-Komposita mit bzw. ohne Fugen-e- finden wie bspw. Stinkdecke vs. Stinkedecke, Tanzmädchen vs. Tanzemädchen usw., die semantisch differenziert sind. Demnach besitzen die interfigierten Bildungen keine restriktiven Artenlesarten, ,sondern vielmehr nicht-restriktive Beschreibungen bestimmter Eigenschaften der Referenten: Stinkedecke bezeichnet weniger einen speziellen Typ von Decke, sondern beschreibt eher einen speziellen Zustand einer einzelnen Instanz; etwa dahingehend, dass die bezeichnete Decke gereinigt werden muss." (Härtl 2015: 161). Damit entspricht die Semantik des verbalen Erstelements vom Typ Stinkedecke der Semantik von Stadienprädikaten im Vergleich zu der generischen Semantik der interfixlosen Komposita (Härtl 2015: 174). Das beschriebene Phänomen scheint $\mathrm{m}$. E. jedoch nur sehr eingeschränkt produktiv zu sein, v.a. da Neubildungen i. d. R. nicht möglich sind (cf. Surfmanöver vs. *Surfemanöver, Brennholz vs. *Brenneholz, Chill-Versuch vs. *Chille-Versuch, Ess-Papier vs. *Esse-Papier). Möglicherweise handelt es sich um (Reste von) regionale(n) oder dialektale(n) Formen, die in komplementärer Distribution zu anderen regionalen Formen stehen wie standard-österr. Blasbalg, Tragtasche (statt bundesdt. Blasebalg bzw. Tragetasche). Die Beispiele vom Typ Stinkedecke stellen allerdings semantisch keinen Widerspruch zu den hier getätigten Ausführungen dar bzw. können sogar umgekehrt als indirekte Evidenz dafür gewertet werden, da feminine Abstrakta aufgrund ihrer formal inhärenten Bieventivität es auch vermögen, Stadien zu prädizieren, z. B. bei Pluralisierung wie bspw.: Untersuchung - Untersuchungen, Berechnung - Berechnungen, Husterei - Hustereien, Abgeschlagenheit - Abgeschlagenheiten, Kälte - Kälten ,Kälteperioden' (zu Details cf. Abschnitt 3.2). Die Semantik von Nominalisierungen ist somit parallel zu der von Härtl (2015) beobachteten Semantik. Aus diesem Grund wäre auch synchron-morphologisch das Morphem -ewie in Tanz-e-mädchen demzufolge besser als Nominalisierungssuffix als als Fugenelement unklarer diachroner Provenienz einzuordnen. 
Genus) größere Relevanz beim Aufbau von morphologischer Komplexität ( $>$ Länge) zukommt, da diese im Gegensatz zu Nullderivationen in der Lage sind, externe Argumente, wie die VP Spulen drehen, zu sich zu nehmen wie bspw. dt. Spulen-drehung vs. *Spulen-dreh (cf. z. B. Alexiadou et al. 2007: 538-541).

Man könnte an dieser Stelle einwenden, dass die gerade dargestellte Reduktion derivationsbzw. flexionsmorphologischer Inhalte aufgrund ihrer Einmaligkeit kein hinreichendes Kriterium für die morphologische Theoriebildung sein könne. Dagegen spricht jedoch, dass sich abermals ein paar Jahrhunderte später, nämlich Ende des 18. Jahrhunderts, vergleichbare Reduktionsprozesse deverbaler Nominalisierungen auf den Stamm beobachten lassen - in diesem Fall mit dem produktiven und nach Demske (2000) seit dem Fnhd. in Inproduktivität begriffenen Suffix -ung und dem seit dem 17. Jahrhundert zunehmend auftretenden (cf. Wilmanns 1896: 531; Demske 2001: 314), Fugen-s-. So wird z. B. Ableitung-s-silbe zu Ableitsilbe, Anmietung-s-vereinbarung zu Anmiet-vereinbarung, Betret-ung-s-verbot $\mathrm{zu}$ Betretverbot (cf. auch Kapitel 2). Die Parallelität beider Vorgänge ist morphologisch nur erklärbar im Rahmen einer Zusammenschau der diachronen Vorgänge, denn dass Reduktions- bzw. Reinterpretationsprozesse (in der Grammatikalisierungstheorie auch als sog. Reanalyse bezeichnet) einen maßgeblichen Anteil an Grammatikalisierungen haben, ist allgemein bekannt. Dass diese jedoch auch wortintern ablaufen können und sogar bei zeitlicher Versetzung sogar wiederholt auftreten können, kann nur so interpretiert werden, dass hier maßgebliche Kräfte zur Kodierung eines morphologisch außerordentlich relevanten Schritts am Werk sind, deren Arbitrarität in Anbetracht der gerade getroffenen Aussagen als außerordentlich fragwürdig erscheinen muss. Im Gegenteil: Der Sprachwandel in Form einer Integration von deverbalen Nominalisierungen mit anschließender Reduktion auf den Stamm ist sogar mehrmals passiert. Im Gegenwartsdeutschen ist die einstige morphologische Parallelität beider Bildungsmuster in Form suffixaler Konkurrenz zuweilen noch belegbar wie bspw. bei Bildetyp/Bildungstyp, Ausrufezeichen/Ausrufungszeichen oder Ablesedatum/Ablesungsdatum.

Auch aus dem Englischen gibt es zusätzliche Evidenz dafür, dass derivierten Nomina eine Schlüsselrolle beim morphosyntaktischen Komplexitätsaufbau der Komposition zukommt: Im Gegenwartsenglischen können produktiv gebildete Suffigierungen nämlich nicht als Erstelemente fungieren, cf. folgende, stichprobenartige Beispiele aus dem British National Corpus ${ }^{18}$ : *faintness feeling, aber feeling of faintness, *foreignness experience, aber experience of foreignness, *nubility certificate, aber certificate of nubility sowie ?compexification strategy, aber strategy of complexification/of making things more complex. Im Gegenwartsdeutschen können die entsprechenden morphologischen Korrelate jedoch allesamt über die Komposition enkodiert werden: cf. Schwächeanfall, Fremdheitserfahrung, Ehefähigkeitszeugnis bzw. Komplexifizierungsstrategie. Auch umgekehrt wäre eine ,wörtliche' Übersetzung des Deutschen Kompositums Verbindungsglied ins Englische nach Auskunft von Muttersprachlern nicht möglich:*connection link. ${ }^{19}$ Die Restriktionen des Englischen zeigen, warum seine

\footnotetext{
${ }^{18}$ Cf. http://corpus.byu.edu/bnc [19.05.2016].

${ }^{19}$ Systematische Proben aus dem Corpus of Contemporary American English (COCA, cf. http://corpus.byu.edu/coca [19.05.2016]) ergaben kaum Belege für die produktiven Suffixe -ness sowie -ity (wie group cohesiveness level, drug awareness programm, network embeddedness theory), wobei sowohl die geringe Belegzahl als auch lexikalische Besonderheiten des fachsprachlichen Gebrauchs (insbesondere aus der Verwaltungssprache) auf sehr eingeschränkte Produktivität schließen lassen.
} 
Komposita weniger morphologisch komplex, d. h. kürzer sind als Komposita des Deutschen: Das Englische scheint hier ererbte Distributionspräferenzen der Komposition fortzusetzen, da sich auch diachron einige Restriktionen bzgl. Nominalisierungen (insbesondere auf -ing) als Kompositionserstelemente feststellen lassen (cf. Kastovsky 2007). Diachron bleibt an dieser Stelle festzustellen, dass der Zusammenfall von Partizip I und ing-Nominalisierungen in der Geschichte des Englischen (cf. z. B. Alexiadou 2013) parallel zur Integration von ingKonstituenten in das Paradigma der Komposition verläuft (cf. Kastovsky 2012). Doch auch syntaktisch sind die Restriktionen synchron motiviert, denn durch eine Nominalisierung würde grammatisch eine OV-Struktur impliziert, welche inkompatibel mit den strukturellen Gesetzmäßigkeiten der linksköpfigen VP des Englischen ist (cf. auch Abschnitt 1.1 zur Rolle der Syntax).

Aus Sicht der Grammatikalisierung für das Deutsche konnte eine Produktivität der Determinativkomposition vom Typ $\mathrm{N}+\mathrm{N}$ nur dann uneingeschränkt zur Entfaltung kommen, sobald auch produktive Nominalisierungen als Erstelemente von Determinativkomposita formal zugelassen wurden. Doch offenbar stellt dies im Sprachwandel, wie diachron bereits am Beispiel des Englischen illustriert wurde, eine morphologische Hürde dar. Als morphosyntaktischen Grund könnte man an dieser Stelle vermuten, dass die getrennten Wortbildungyprozesse Determinativkomposition und synthetische Komposition ( $>$ Suffigierung) durch die Integration von Nominalisierungen als Erstelemente einander kreuzen. Dadurch geht die Distinktheit beider Wortbildungsmuster verloren und führt zu strukturellen Ambiguitäten (wie in Abschnitt 2.2 bereits am Beispiel des Typs Wetterbeobachtung deutlich wurde), die diachron das Sprachsystem erst schrittweise überwindet und die synchron jedoch das Potenzial zu neuer Strukturbildung birgt (cf. Abschnitt 6.1 zur prospektiven Betrachtung der Komposition).

Vor dem Hintergrund der gerade zusammengestellten Befunde in Bezug auf verbale Bestandteile kann nun auch die retrospektive Frage eines möglichen, analytischen „Vorläufers“ der erst im Mhd. feststellbaren $\mathrm{V}+\mathrm{N}-$ Komposition beantwortet werden, denn auf eine mögliche Konkurrenz zwischen Part. I und V+N-Komposita bzw. Komposita mit deverbalem Abstraktum als Erstglied weist bereits Grimm (1837: 67) hin. Im Nhd. verweist dabei das Partizip Präsens auf das interne Argument (gegessene Suppe zu ,x hat Suppe gegessen, Suppe wurde gegessen'), dagegen verweist das Partizip Perfekt auf das externe Argument (wie in essende Mutter zu ,die Mutter isst'). Man könnte daraus folgern, dass solche Konstruktionen auch vor Aufkommen der Komposition im Deutschen vorherrschten, denn im Mhd. konnten im Gegensatz zum Nhd. attributiv verwendete Partizipia I auch passivisch verwendet werden, was die Vermutung nährt, dass es sich bei diesen um verbale ,Vorläufer' der späteren verbalen Erstelemente gehandelt haben könnte. Ein solches System liegt heute bspw. im determinativkompositionslosen Russischen (cf. Kapitel 2) sowie dem Ungarischen (Typ: ír-ó-asztal wörtl. ,schreibender Tisch', dt. Schreibtisch) vor. Cf. hierzu die diesbezüglich uninterpretiert gebliebene Feststellung von Paul (1881/2007: 309):

Das Verhältnis zwischen attributivem Part. [Präsens] und Subst. kann weniger eindeutig sein, indem z. B. Beziehungen verschiedener Art zwischen Verbum und Nominalbegriff ausgedrückt werden, für die eine jüngere Sprachstufe bzw. andere Sprache jeweils gesonderte Ausdrucksformen gesonderter Art gefunden haben. In einigen der folgenden Fälle ließe sich das Part. gerundiv oder als Gerundium ,übersetzen"; die beigefügten nhd. Entsprechungen stellen aber nur eine Möglichkeit der Wiedergabe dar. Manche Verbindungen werden bevorzugt in mehrdeutigen Verbindungen gebraucht, andere vereinzelt: [...] der minnend unsin (,der Wahnsinn des 
Liebens') [...], in sterbender nôt (,in der Not des Sterbens') [...], schamende arbeit (,Arbeit, bei der man Scham empfindet") [...], ein lebender Tag (,ein Tag des Lebens, an dem ich lebe') $[\ldots]$.

Grimm (1837: 64-71) zufolge nimmt die passivische Verwendungsmöglichkeit des Part. Präsens im Mhd. sogar kurzfristig zu, was dazu passt, da das Mhd. im Vergleich zu späteren Sprachstufen noch seltene Fälle von V+N-Komposita aufweist, d. h. dieses Muster gerade über die -e-Reduktion ausbaut, umgekehrt jedoch die heutigen Präsenspartizipien ausschließlich agentivisch interpretierbar sind. D. h. beide Prozesse verlaufen spiegelbildlich. Das Bairische bspw. scheint diesen alten Sprachzustand noch in einigen Fällen konserviert zu haben. So bspw. bei sitzader Tag wörtlich, sitzender Tag, Tag an dem man sitzt' in der Frage Hast Du heit dein'n sitzaden Tag? ,Hast Du heute deinen Sitz-Tag?'. ${ }^{20}$ Auch aus sprachtypologischen Erwägungen (cf. Ungarisch) wäre die vorliegende Interpretation plausibel. Im nächsten Abschnitt soll für das Deutsche nun die Ebene der Synchronie und der Diachronie aufeinander bezogen werden.

\section{Zusammenführung der synchronen und diachronen Befunde}

Die Unterscheidung von Synchronie und Diachronie zeigt, dass die jeweils beobachtbaren formalen Restriktionen in Bezug auf das Erstelement nicht dieselben sind. Damit liegt eine Diskontinuität im Sinne einer Dynamik vor, welche aufgrund des Aufbaus semantischer Differenz zu analytischen Strukturen im Gegenwartsdeutschen und diachroner morphologischer Entwicklungen (Reduktions- und Neutralisationsprozesse beim Erstelement) mit dem Instrumentarium der Grammatikalisierungstheorie in Einklang gebracht werden kann. Zunächst sollen hierzu die formalen Restriktionen und ihre sich daraus ergebenden weiteren formalen Konsequenzen, darauf die semantisch-konzeptuellen Konsequenzen für den Bildungstyp der Komposition ausgeführt werden.

\subsection{Formale Entwicklung im Sinne einer Grammatikalisierung}

Aus der Sprachgeschichte lassen sich zwei maßgebliche Veränderungen feststellen, die einen Sprachwandel im Sinne einer Grammatikalisierung plausibel machen: a) die allmähliche Erweiterung der Wortarten des Erstelements (von nominal im weiten Sinne zu nicht-nominal) sowie b) die Integration jeweils produktiv derivierter deverbaler und deadjektivischer Abstrakta, was im Ahd. noch nicht möglich war und über die Reduktion der Suffixe von deverbalen Abstraktnominalisierungen ( $-e$ im Mhd. sowie -ung und zunehmend mit Fugenelement $-s-$ $\mathrm{ab}$ fnhd. Zeit) auf den Stamm verlief (cf. Abschnitt 5.3). Durch den Wegfall jedweder Markierung (wie Suffixe, Flexionsmorpheme) kommt es zur einer Neutralisation der Wortartinforma-

\footnotetext{
${ }^{20}$ Eine noch üblichere Form wäre die verbale Periphrase ausschließlich mit nominalisiertem Partizip Hast Du heit dein'n Sitzaden? Das Partizip Präsens kodiert hier zusammen mit dem Finitum haben (wie auch in an Lachaden ,einen Lachenden', an Rehraden ,einen Weinenden', an Blearaden ,einen Plärrenden, Brüllenden' usw. haben) iterative Habitualität, aus der sich übereinzelsprachlich bevorzugt die Generizität entwickelt. Iterativität besteht stets im Allgemeinen aus der Merkmalskombination von Perfektivität und Imperfektivität (cf. Dressler 1968). Die Nominalisierung des maskulinen Partizips besteht konzeptuell aus dem Vordergrundieren eines perfektiven Abschnitts (zählbares Maskulinum) bei Hintergrundierung der imperfektiven, da in Form des Partizip Präsens kodierten Verbalität.
} 
tion, was in der Grammatikalisierungsterminologie mit „Dekategorisierung” (Verlust der Wortartinformation) bezeichnet wird. Entsprechende Bildungen des Gegenwartsdeutschen sind daher, so keine Lexikalisierung oder Usualisierung semantisch einschränkend wirkt bzw. durch entsprechende Aktualisierungskontexte monosemiert wird, semantisch unterspezifiziert (sog. root-Semantik). Das bei der Komposition sich grammatikalisierende Formmerkmal ist das der Generizität (auch: Typisierung oder klassifikatorische Semantik, cf. Schlücker 2014) wie in Schwimm-kerze ,Kerze die (per se) schwimmt/schwimmen kann', Schwarzlicht ,Licht, das (inhärent) schwarz ist' usw. Damit stehen komponierte Formen in formaler wie semantischer Hinsicht in Opposition zu analytischen Konstruktionen (wie schwimmende Kerze ,Kerze, die (gerade) schwimmt', schwarzes Licht ,Licht, das gerade schwarz ist/schwarz geworden ist' usw.

Obgleich empirische Untersuchungen an historischem Sprachmaterial zur Beseitigung empirischer Lücken weiterhin unabdingbar sind (cf. auch Abschnitt 1.2), lassen sich vor dem Hintergrund der bereits in der Literatur dokumentierten Befunde dennoch vorsichtige Rückschlüsse hinsichtlich der Datierung der Umbrüche ziehen: Historisch sind deverbale und deadjektivische Nominalisierungen (genauer: die produktiven auf -heit/-keit und -ung sowie schaft) nicht vor mhd. Zeit, was eigene Recherchen ergaben, als Erstelemente in Erscheinung getreten. Im Ahd. waren Nominalisierungen mit den zu dieser Sprachstufe entsprechenden, produktiven Suffixen also nicht als Erstelemente von Komposita realisierbar, sondern wurden analytisch realisiert. In mhd. Zeit sind auch eine erhebliche Anzahl an „Schwankungen“ von Komposita mit bzw. ohne -e- zu finden (wie in schab-mezzer/schabe-mezzer, slaht-hus/slahtehus, cf. Abschnitt 5.3), was als Reduktion eines Suffixes einer im Erstelement zugrundeliegenden Nominalisierung gefasst wurde. Die entsprechenden Bildungen werden dadurch auf den Stamm reduziert, was, neben der bereits im Ahd. dokumentierten Integration nullderivierter Deverbalia (wie ahd. blas ,Luftstoß'), aus syntaktischen Gründen (cf. Abschnitt 5.3) v. a. durch die ehemals explizit derivierten Nominalisierungen, in der Folge auch die Integration von $\mathrm{V}^{0}$ und später auch $\mathrm{X}^{0}$ (sog. Phrasenkomposita in Form von metasprachlichen Einheiten wie das ich-will-nicht-ins-Bett-Kind) mitbeförderte und somit eine gegenwartssprachlich zwar zunächst wortartengeleitete, aber durch die Univerbierung nun eine wortartenneutralisierte Interpretation der Erstelemente nahe legt. Parallel hierzu ist die Reduktion der prominenten ung-Nominalisierungen (in fnhd. Zeit mehrheitlich noch ohne, ab dem 18. Jh. zunehmend mit -s-Fuge, cf. Werner 2016) zu stellen, die ein paar Jahrhunderte später ebenfalls auf den Stamm reduziert werden (Typ: Anmietungsvereinbarung $\rightarrow$ Anmietvereinbarung). Letztere Bildungen sind aus gegenwartssprachlicher, nicht aber aus Sicht des 18. Jh. ganz selbstverständlich. Als Beweis können auch Dubletten zu nicht-nativen Nominalisierungen vs. Stammkomposita dienen wie etwa Inhalationsspray/Inhalierspray. Eine Bildung wie bspw. Brechbohnen ist zwar durch Usualisierung festgelegt auf die Bedeutung ,gebrochene Bohnen', ohne Usualisierung jedoch wären auch andere Interpretationen denkbar wie etwa ,brechende Bohnen', ,Bohnen von einer Person namens Brech', ,Bohnen in der Art einer Person namens Brech', brechbare Bohnen' u. a. Der dynamische Prozess der Reduktion auf die Wurzel ist gegenwärtig noch nicht abgeschlossen, da immer noch Abstraktnominalisierungen als Erstelemente fungieren können (cf. Umgehungsstraße, Schönheitskönigin).

Damit lässt sich nach dem ersten Reduktionsschritt mhd. Abstrakta im frühen Nhd. eine abermalige Reduktion im Bereich des Erstelements konstatieren. Alle produktiven und dever- 
balen Nominalisierungen, d. h. die auf -ung, -erei und die komplexeste Schicht der erSuffigierungen (cf. Werner 2012; cf. auch Kapitel 5), nämlich die Nomina acti (wie Hopser, Juchzer) tendieren, so überhaupt als Erstelemente realisierbar (cf. *Lacherei-Versuch; *Lachen(s)versuch), zum Gegenwartsdeutschen hin zunehmend zur Reduktion auf die Wurzel (engl. root). Dieselbe Restriktion ist zwar nicht bei deadjektivischen Nominalisierungen zu beobachten (wie Wahrheits-tick $\rightarrow *$ Wahr-tick, Coolheits-Geste $\rightarrow *$ Coolgeste), jedoch bei derivierten Adjektiven: *Farbigpapier, *Salzigwasser, *Künstlichhonig, aber Farbpapier, Salzwasser, Kunsthonig. Bei deadjektivischer/departizipialer Nominalisierungen bleibt das Erstelement hingegen, wie zu erwarten bei -heit-Nominalisierungen, unreduziert, nimmt allerdings stets das Fugenelement -s- zu sich (wie bei Schönheit-s-/*Schönheit-fleck, Benommenheit-s-/*Benommenheit-symptom, cf. Kapitel 5). Vollmotivierte departizipiale Zustandsnominalisierungen (wie Verliebtheit, Verschwiegenheit, Verwirrtheit, cf. Bücking 2012) werden als Erstelemente also nicht auf den Stamm reduziert, cf. Verlassenheits-gefühl $\rightarrow$ *Verlass(en)-gefühl, Verliebtheits-zustand $\rightarrow * V e r l i e b(t)$-zustand, Bedrücktheits-laune $\rightarrow$ *Bedrück(t)-laune. Die departizipialen Nominalisierungen sind somit diesbzgl. morphologisch analog zu den deadjektivischen, nicht aber zu den deverbalen.

Aus Sicht des Sprachwandels wäre langfristig auch die Frage interessant, ob auf Basis des bisher Dargestellten auch künftige Entwicklungen u. U. - wissenschaftstheoretisch natürlich nur in nicht-teleologischer Weise - prognostiziert werden könnten. Konkret würde dies bedeuten, inwieweit langfristig die departizipialen Nominalisierungen in Analogie zu dem gegenwärtig restriktionslosesten Nominalisierungsmuster für Verben, dem substantivierten Infinitiv, gleichziehen und statt der Suffigierung mit -heit den Infinitiv bevorzugen (so z. B. Verliebtheit $\rightarrow$ (das) Verliebtsein, Abgeschlagenheit $\rightarrow$ (das) Abgeschlagensein, Verwirrtheit $\rightarrow$ (das) Verwirrtsein). Da produktiv gebildete substantivierte Infinitive bis dato nicht als Erstelemente von Komposita fungieren können (cf. Abschnitt 4.2), wäre an dieser Stelle die Frage spannend, wie das Sprachsystem sich weiterentwickeln wird. Gegenwärtig liegen zwei Muster vor, welche beide gleichermaßen plausibel wären: entweder würden bereits vorhandene Muster mit Sein-s-x (wie Daseinsfrage) ausgebaut oder aber der Wortbildungstyp Komposition würde für diese Bildungen nicht weiter bestehen und die Bildungen würden analytisch, d. h. syntaktisch kodiert. Erste Internetrecherchen lassen eher die zweite Variante als möglich erscheinen (wie Zustand des Verliebtseins statt?Verliebtsein(s)zustand oder Problematik des Verlassenwordenseins statt?Verlassenwordensein(s)-gefühl). Da es sich bei den Infinitivnominalisierungen wie bei Abstrakta typisch um inhärent generische Propositionsnominalisierungen handelt und dem definiten Artikel hierbei keine bekanntheitherstellende Funktion im strengen Sinne (Kernfunktion der Definitheit) zukommt, sondern im Gegenwartsdeutschen auch generische Semantik bereitstellen kann, wären hier, so kann man vermuten, morphosyntaktisch komplexe und weitreichende Umkodierungsprozesse möglich, welche in Analogie zur Entwicklung der linksköpfigen Struktur der Nominalphrase wären (cf. auch Abschnitt $5.3)$.

\subsection{Zur grammatischen Semantik des Erstelements}

Mit der schrittweise hergestellten Beseitigung von Restriktionen ist eine Neutralisation grammatischer Semantik verbunden (cf. Kapitel 5) mit der Folge, dass nahezu alle quantifi- 
zierenden Morpheme (Suffixe, Flexive) auf der Position des Erstelements blockiert sind. Die Restriktionen umfassen die gesamte Partizipialmorphologie (so nicht nominalisiert wie in Lernenden-Seminar), stative Adjektivsuffixe (wie -lich, -haft, -sam, cf. Vogel 1996: Abschnitt 3.4), transnumerale nominalisierte Infinitive sowie die substantivische Derivation, mit Ausnahme von -ung- (zur fraglichen Produktivität cf. Demske 2000) sowie -heit/-(ig)keitNominalisierungen, die stets ein Fugenelement $-s$ - erhalten (cf. hierzu nächster Absatz). Abgeleitete Wörter werden im Regelfall also auf die Root (,lexikalisches Konzept ohne grammatische Semantik und Wortarteneintrag, d. h. Unterspezifikation", zum Überblick cf. Olsen 2012) reduziert, was im Rahmen der Grammatikalisierungstheorie mit dem Terminus Kondensation bezeichnet wird.

Einzig deadjektivische Nominalisierungen, die durch produktive Suffixe genus-quantifiziert enkodiert werden (cf. Abschnitt 3.2), sowie historisch die -ung-Nominalisierung werden bei der Verwendung als Erstelemente nicht reduziert, treten aber obligatorisch mit Fugenelement $-s$ - auf. Dem Fugenelement kommt dabei die Rolle eines ,Quantifizierungsneutralisators" zu (cf. Werner 2016), da dieses einerseits die generisch-propositionale Semantik des femininen Abstraktums erhält (Typ: Schönheit-s-königin ,Königin (nach dem Prinzip) der Schönheit”) und das Erstelement andererseits, auch im Sinne von Fuhrhops (z. B. 2000) Morphologisierung, für weitere morphologische Prozesse (hier: der Komposition) anschlussfähig gemacht wird (cf. Kapitel 4 und 5). So wird beim Vorliegen eines deverbalen Kopfs verhindert, dass die Nominalisierung als Argument interpretiert wird (wie beim Typ [[Minderheiten-wahl]recht] ,Recht, gemäß dem Minderheiten wählen').

Die semantische Folge dieser diachron feststellbaren Paradigmatisierung im Zuge der Grammatikalisierung ist, dass die semantische Spannbreite von Komposita ohne spezifische Kontextualisierung diachron kontinuierlich erweitert wurde. So ist zwar ein Kompositum wie Backerbse durch Usualisierung auf die Bedeutung ,Erbsen aus Gebackenem/Back-" festgelegt. Ohne eine spezifische Kontextualisierung jedoch können je nach Aktualisierungskontext, der einzelfallspezifisch i. d. R. durch Frequenz konventionalisiert werden und so die Semantik verfestigen kann, formal unterschiedliche Interpretationen aufgerufen werden, so bspw. ,gebackene Erbsen', ,(zu) backende Erbsen' und weitere. Auch zu einer Bildung wie Kochschinken wären weitere Interpretationen formal möglich wie etwa ,gekochter Schinken', ,(zu) kochender Schinken', ,kochbarer Schinken', ,Schinken des Kochs', ,Schinken nach Art des Kochs' sowie sogar mit Eigennamen ,Schinken (nach Art) von einer Person namens Koch' (cf. auch Abschnitt 5.2).

Anhand der parallelen Verfügbarkeit unterschiedlicher Paraphrasen (s. o.) bei Kontextfreiheit kann auch gezeigt werden, dass die Position des Erstelements eine wortartenspezifische Quantifizierung neutralisiert. Allerdings könnte man semantisch einwenden, dass diese stattdessen eine wortartenunabhängige Homogenität besitzen würde; dies entspräche - kurz gefasst - bei Substantiven der Semantik von Massennomina (cf. z. B. Rijkhoff 1992), bei Adjektiven der Stativität sowie bei Verben der Imperfektivität/Atelizität (cf. z. B. Vogel 1996). Für die Interpretation zugunsten von Homogenität spräche einmal, dass Massennomina niemals Fugenelemente zu sich nehmen (cf. *Ölslampe, *Fettsauge, *Sandskorn), d. h. nur zählbare Substantive können mit Fugenelementen einhergehen (wie Hühnerstall, Häuserzeile, Bilderrahmen). Darüber hinaus können Eigennamen als Erstelemente fungieren und eine ,Art-von"Semantik auslösen (wie Klimt-Espressotasse ,Espressotasse in der Art von Klimt', cf. auch 
Abschnitt 5.2). Dieses Phänomen ist aus der Sprachtypologie bereits bekannt, so bspw. aus dem Finnischen. Dort können Eigennamen neben ihrer herkömmlichen Verwendung (z. B. als Subjekt wie in (1)) auch im Partitiv verwendet werden (cf. Kapitel 2; beide aus Balles 2004: 31f.):

\section{(1) Se on Lisa , ,Das ist Lisa.'}

(2) Se on Lisa- $a_{\text {Part }} \quad$ wörtl. ,Das ist lis-isch', d. h. ,Das ist typisch für Lisa.'

Da Partitive immer Homogenität implizieren, jedoch Eigennamen semantisch aufgrund unikaler Referenz immer als inhärent zählbare Nomina gelten, entsteht durch ihre Setzung in den Partitiv ein grammatischer Widerspruch, der so gelöst wird, dass die Eigennamen die ,Artvon'-Interpretation annehmen. Übertragen auf die Komposition des Deutschen wäre vor dem dargestellten Hintergrund die Ablehnung der Quantifizierungsneutralisations-Hypothese und stattdessen die Annahme einer Homogenität-Hypothese zunächst naheliegend. Allerdings lassen sich aus den dargestellten Distributionen und Restriktionen Konklusionen ableiten, die die letzte Annahme als unplausibel erscheinen lassen. Denn Eigennamen werden im Deutschen nicht nur in der ,Art-von'-Bedeutung realisiert, sondern können kontext- und lexikalisierungs- bzw. usualisierungsfrei immer auch die ursprüngliche, possessive Semantik realisieren, cf. etwa Mozartstück, Stück von Mozart' sowie ,Stück in der Art von Mozart'; Letzteres gilt insbesondere dann, wenn die possessive Lesart kontextuell ausgeschlossen ist wie bspw. in der Äußerung Hans hat ein Mozartstück komponiert. Es sind also formal immer mehrere Interpretationen verfügbar. Darüber hinaus sind die Fugenelemente nicht bei allen zählbaren Substantiven obligatorisch oder morphologisch systematisch distribuiert (cf. synchron Vogelnest, aber *Vögelnest; Handymast, aber *Handysmast; Fuchsbandwurm, aber *Füchsebandwurm), selbst dann nicht, wenn die Singularform bspw. aus metaphysischen Gründen, naheliegen würde wie bei Hühnerei. Fugenelemente lassen sich historischstrukturell nicht an eine Singular- bzw. Pluralsemantik anbinden (cf. Henzen 1965; Wegener 2005). Umgekehrt können im Gegenwartsdeutschen zählbare Nomina (wie Erdbeere, Ananas) in Komposita die homogene Semantik annehmen (wie bei Erdbeer-Shake, AnanasGeschmack), wobei diese nicht immer vorliegen muss (cf. Erdbeer-Feld, Ananas-Plantage), während Stoffnomina homogen (wie in Holzregal ,Regal aus Holz') und inhomogen (wie in Weinkiste ,Kiste für Wein(e)') interpretierbar sind. Bereits früh (Augst 1975: 103) wurde in diesem Zusammenhang festgestellt, dass die Mehrzahl der Komposita, nämlich mehr als zwei Drittel (cf. Krott et al. 2007: 27), im Gegenwartsdeutschen keine Fugenelemente besitzt. Vergleichbare Ergebnisse konnten jüngst durch psycholinguistische Experimente zum kindlichen Spracherwerb (cf. Dressler et al. 2010) und zur Erwachsenensprache (cf. Neef/Borgwaldt 2012) erzielt werden. Die Ermittlung von diachronen Fugenelement-Diskontinuitäten muss neben der Erhebung von Nicht-Belegbarem (cf. Kapitel 1) somit einerseits die Unterscheidung von grammatisch motivierten Bildungen (wie produktiven Nominalisierungen) vs. lexikalisierten Bildungen und andererseits mögliche Reduktionsprozesse (wie die in Abschnitt 6.3 dargestellten) in den Blick nehmen, da grammatische Strukturbildung bekanntlich stark musterbildend (i. S. v. paradigmatisch, cf. Abschnitt 1.2) ist und es bei Nichtberücksichtigung dieser beiden Sprachrealitäten (i. S. v. „belegbar“" vs. „nicht-belegbar“) in der empirischen 
Arbeit zu quantitativen „Verzerrungen“ kommen kann, auch indem der Status der erhobenen Belege in Bezug auf grammatische vs. lexikalische Repräsentativität unklar bleibt.

Um bei der Suche nach repräsentativen Belegen der Gefahr einer Auswahl möglicher Lexikalisierungen $\mathrm{zu}$ entgehen und gleichzeitig gegenwartssprachlich valide Beispiele zu erhalten, lässt sich die Semantik von Erstelementen anhand der in Abschnitt 5.1 dargestellten Distributionen allerdings prüfen: Nominalisierte Infinitive sind nicht-pluralfähige, $d . h$. homogene Substantive (Typ: das Chatten - *die Chatten), die jedoch produktiv gebildet nicht als Erstelemente von Komposita fungieren können wie *Schwimmensbad, *Hustensattacke (zum nominalisierten Infinitiv das Husten), *Lachensanfall, sondern einen morphologischen Reduktionsprozess (Typ: Schwimm-bad, Hust-attacke, Lach-anfall) durchlaufen. Wären Erstelemente semantisch homogen, so müssten nominalisierte Infinitive als Erstelemente von Komposita morphologisch möglich sein. Dies ist jedoch nicht der Fall: Auch hinsichtlich Stativität markierte Adjektive können nicht als Erstelemente realisiert werden. Umgekehrt liegen stammreduzierten Komposita verschiedene Bedeutungen zugrunde (wie Jodel-Lautstärke ,Lautstärke des Jodelns', ,Lautstärke eines (einzelnen) Jodlers' (Nomen acti) und ,Lautstärke der Jodlerei', ,Lautstärke von (Herum-)Gejodele', cf. Abschnitt 3.2, 4 und 5.3. Aus den genannten Gründen erscheint die Annahme einer root-Semantik, d. h. eine semantische und morphologische Unterspezifikation des Erstelements, plausibel. Dass hier auch kein ggf. impliziter Konversionsprozess zugrunde liegt, in dem Sinne, dass alle Konstituenten in eine spezifische Wortart (z. B. Substantiv) überführt würden, ist daran zu ersehen, dass der Stamm wortartenneutral, so auch in Bezug auf seine vielfältige semantische Paraphrasierbarkeit bei Kontextfreiheit, ist und dass grammatisch markierte Nomina in Form von Nominalisierungen spezifischen Selektionsmechanismen, auch in Bezug auf die Interfigierung, bzw. Reduktionsmustern unterliegen (cf. auch Abschnitt 4.2).

\section{$7 \quad$ Fazit und Ausblick}

Der Artikel widmete sich der diachronen Komplexitätserhöhung der substantivischen Determinativkomposition und damit der Frage, warum Substantive im Verlauf der deutschen Sprachgeschichte immer länger wurden (erstmals thematisiert von Wurzel 1996). In diesem Beitrag wurde unter der Annahme eines Sprachwandelprozesses im Sinne einer Grammatikalisierung argumentiert, dass bei der Determinativkomposition weniger dem Zweit- als vielmehr dem Erstelement eine tragende Rolle vom Aufbau grammatischer Semantik (in Form der Generizität, cf. Kapitel 4) zukommt. Diese kann v. a. über den Parameter der Dekategorisierung im Sinne einer Wortartenneutralisation des Erstelements bei paralleler Integration derivierter Abstraktnominalisierungen (cf. Abschnitt 5.2, 5.3 und 6.1) charakterisiert werden. So werden zur Bildung von Determinativkomposita morphosyntaktisch alle Wortarten mit lexikalischem Inhalt oder „lexifizierbarem” Inhalt (i. S. v. metasprachlichen Einheiten, cf. Wiese 1996, Typ: ein ,,ich-will-nicht-ins-Bett"-Kind) selegiert, die durch die Setzung als Erstelemente hinsichtlich ihres Wortarten- und Quantifizierungseintrags neutralisiert werden.

Auch die kontroverse Datierung der Komposition („Ahd.“ vs. „Fnhd.“, cf. Abschnitt 2.2) kann im Rahmen der Theorie über sog. Layering (Überlagerung alter und neuer Formen) motiviert werden: Bis in fnhd. Zeit überlagern sich demnach alte Formen (in Form des pränominalen Genitivattributes) und Komposita, wobei zwischen beiden Formen kein semantischer 
Unterschied besteht (cf. Abschnitt 5.1). Unter der Annahme, dass verschiedene Enkodierungsformen während der Grammatikalisierung miteinander konkurrieren, wobei schließlich eine präferiert wird (sog. Spezialisierung), kann hier aus Sicht des Erstelements, insbesondere die in vielen anderen Sprachen vorherrschende, morphologische „Alternative” in Form der Adjektivattribute, d. h. die diachrone Entwicklung des einst reichhaltigen suffixalen Adjektivmarker (cf. Kapitel 5) gestellt werden, deren Abbau zum Nhd. hin zwar konstatiert, bislang aber eine Interpretation seiner Existenz schuldig geblieben ist. Unter der Annahme einer Grammatikalisierung der Komposition ergeben sich also auch in Bezug auf verwandte Kodierungsmöglichkeiten künftige Perspektiven für die Wortbildungsforschung, insbesondere vor dem Hintergrund sprachlicher Variation (cf. Abschnitt 2.2).

Mit dem Aufbau morphologischer Komplexität war neben der schrittweisen Wortartenerweiterung (hier als Dekategorisierung gefasst) auch die Reduktion von Nominalisierungen auf ihren Stamm verbunden (eine wortinterne Kondensation des Erstelements, cf. Abschnitt 5.2). Gerade dieser letzte Schritt fand mehrmals, d. h. in verschiedenen Sprachstufen statt (im Mhd. sowie im frühen Nhd., cf. Abschnitt 5.3). Demnach hat die Integration von insgesamt unterschiedlichen, aber in der jeweiligen Sprachstufe produktiven Suffigierungsmustern in die Position des Erstelements für die Deverbalia jeweils zu Reduktionen geführt, so dass die übriggebliebenen Derivationsbasen als Wurzeln reinterpretierbar und für weitere morphologische Anschlussprozesse verfügbar gemacht wurden, wobei mögliche weitere sprachdynamische Momente bereits jetzt aufgrund interner Systemkonfiguration angelegt sind (cf. Kapitel 6). Die Tatsache, dass gerade Nominalisierungen so komplex in das primäre $\mathrm{N}+\mathrm{N}-$ Muster der Komposition zu integrieren sind, erscheint auf den ersten Blick paradox, kann allerdings mit dem hohen Grad an Grammatikalität der Nominalisierungen erklärt werden: Sie sind genusmarkiert im Nhd. (Wegener 2000) und können semantisch in Form von Quantifizierung gefasst werden, wobei der Kollektivität von femininen und neutralen Abstrakta die komplexeste Semantik aufgrund von grammatikalisierter Propositionalität zukommt (cf. Abschnitt 2.2, 5.3 und 6). Feminine und neutrale Abstraktnominalisierungen sind somit semantisch als die „Krone der Schöpfung“ innerhalb der nominalen Grammatik zu bezeichnen.

Auch bei der Entwicklungslogik der Komposition im Deutschen fungieren Reduktions- bzw. Reinterpretationsprozesse als Scharnier von Sprachwandelprozessen, so auch im vorliegenden Fall beim Erstelement, wobei bislang v. a. die historische Rückführbarkeit auf nominale Flexive, insbesondere im Hinblick auf einen Zusammenhang mit der Interfigierung, im Fokus der Aufmerksamkeit stand. Wie im vorliegenden Beitrag argumentiert wurde, ist jedoch die Suffigierung bei Erstelementen von Komposita grammatisch aufgrund deren inhärenter Grammatikalisiertheit von größerer Bedeutung für den Aufbau komplexer grammatischer Strukturbildung. Tatsächlich ist die bis heute bestehende Integration von produktiv derivierten, deadjektivischen Abstrakta (Typ: Schönheit-s-königin) in diesen Zusammenhang zu stellen, während die deverbalen Abstraktsuffigierungen, in mhd. Zeit in Form der -e-Abstrakta, und auch das seit fnhd. Zeit unproduktiv werdende Suffix -ung (cf. Demske 2000) zunehmend auf den Stamm reduziert werden. Dies geschieht im Bereich der Nominalisierungen, d. h. auf Wortebene, parallel zur Produktivwerdung des substantivierten Infinitivs. Die Reduktion unterhalb der Wortebene entspricht damit gleichzeitig einer Quantifizierungsneutralisation.

Da das Deutsche als Vertreter einer flektierenden Sprache über eine grammatikalisierte Nomen-/Verb-Distinktion verfügt (cf. Vogel 1996), stellt die Erzeugung von kategorialer und 
morphosyntaktischer Unmarkiertheit (im Sinne von einer Wurzel/root) womöglich einen höheren prozessualen Aufwand für die Interpretation dar, so kann man folgern, während in flexionsarmen bzw. isolierenden Sprachen solche Umformungsprozesse nicht auf wortinterner, d. h. morphologischer Ebene, sondern vermehrt auf wortexterner, d. h. syntaktischer Ebene (cf. z. B. Englisch *faintness feeling, aber feeling of faintness, cf. Abschnitt 5.3) herzustellen sind. Für flektierende Sprachen entspricht der Abbau von Markierungen einem koverten, strukturell-konfigurationalen Komplexitätsaufbau im selben Maße wie der Aufbau einer derivativen Struktur (z. B. durch ad-hoc-Bildung von Affixoidbildungen) bei isolierenden Sprachen einen höheren prozessualen Aufwand darstellt und damit die Annahme eines Aufbaus von morphosyntaktischer Komplexität plausibel macht. Damit wäre das Ergebnis (hier: rootKomposition) übereinzelsprachlich zwar dasselbe, nicht jedoch der Weg, d. h. der grammatische Prozess dorthin.

Sprachliche Elemente an der Schnittstelle der N-/V-Distinktion, nämlich das Gerund und das Gerundiv, scheinen im morphologischen Kreislauf von Analyse und Synthese eine relevante Rolle für den Umbau von Markiertheitsverhältnissen zu spielen (cf. Abschnitt 5.3). Inwieweit daraus u. U. auf einen zyklisch ablaufenden, kategorialen Synkretismus zu schließen ist, bei dem die (Des-)Integration dieser Formen (cf. englische ing-Form bzw. mhd. Part. I vs. Nominalisierung in Abschnitt 5.3) eine entscheidende Rolle für den Aufbau von Kompositionsstrukturen (Etablierung von synthetischen Strukturen mit Abbau von Markierungen wie im Deutschen, cf. Kapitel 5) bzw. deren Abbau (analytische Strukturen wie im Russischen) darstellt (cf. auch Kapitel 4-6), müssen weitere, insbesondere kontrastive Forschungen zeigen.

Vor dem Hintergrund eines u. U. zyklischen Sprachwandels lässt sich für künftige Forschungsperspektiven festhalten, dass der Grad der Grammatikalität/Grammatikalisiertheit, was eng mit der Produktivität von Suffixen (d. h. deren Fähigkeit zur wortartenspezifischen Reihenbildung in Sprachen mit ausgeprägter N-V-Distinktion bzw. wenig wortartspezifischer Reihenbildung wie im Deutschen, in Sprachen mit geringer N-/V-Distinktion wie im Englischen) verknüpft ist, von entscheidender Bedeutung für die Integration in bereits vorhandene Kompositionsstrukturen ist. Denn durch die spezifische Integration derivierter Konstituenten in das Muster der Komposition wird - wie im Falle der Determinativkomposition des Deutschen - eine Erweiterung des Paradigmas des Erstelements zugunsten von markierten Einheiten eingeleitet, was, da Suffixe stets quantifizieren, aus mereologisch-morphologischer Sicht auch semantisch gelöst werden muss. Dies geschieht entweder durch sekundäre Etablierung eines weiteren Morphems zur Neutralisierung der Suffixsemantik, wie im Falle der sekundären Etablierung des Fugenelements -s- bei Feminina (Typ Schönheit-s-x), oder aber durch Reduktion der Nominalisierungen auf den Stamm, der damit zur Wurzel (engl. root) wird (Typ: mhd. slahte-hus, Schlachtungshaus' $\rightarrow$ slaht-hus, Schlachthaus'). Im Gegenwartsdeutschen zeichnet sich - in Analogie zu den grammatischen, produktiv gebildeten Nominalisierungen - eine Bifurkation ab: Während desubstantivische und deadjektivische Abstraktnominalisierungen zusätzlich interfigieren, werden Deverbalia diachron auf die Wurzel reduziert (wie Betretungsverbot $\rightarrow$ Betretverbot). Unter der Annahme eines möglicherweise zyklischen Sprachwandels dürften beim Zerfall der Komposition in analytische Strukturen insbesondere (de)verbale Konstituenten bedeutsam sein, die entweder als deverbale Abstrakta ( $>$ Gerund) v. a. in Form von Genitiv-/Präpositionalattributen (wie in den romanischen Sprachen, cf. frz. jeu d'amour bzw. älteres Nhd. der Liebe Spiel) realisiert würden oder aber als ,verbales" At- 
tribut ( $>$ Gerundiv) in Form von deadjektivischen bzw. partizipialen Attributen (Typ: lesende Lampe wie bspw. im Ungarischen, cf. Abschnitt 5.1 und 5.3). Die Präferenz zugunsten der jeweiligen Parametersetzung („Gerund” vs. „Gerundiv”) dürfte - prospektiv für weitere Forschung - nicht zuletzt mit morphosyntaktischen Einflussfaktoren wie der VO- vs. OVSerialisierung, des Wortarteninventars sowie der Grammatikalisiertheit eines Artikelsystems (hypo- vs. hyperdeterminierend) in Verbindung stehen und durch deren Berücksichtigung in der historischen Wortbildungsforschung auch in Zukunft vielversprechende Ergebnisse zu erwarten sind.

\section{Literatur}

Ackema, Peter/Neeleman, Ad (2004): Beyond Morphology: Interface Conditions on Word Formation. Oxford: Oxford University Press.

Alexiadou, Artemis/Haegeman, Liliane/Stavrou, Melita (2007): Noun Phrase in the Generative Perspective. Berlin/New York: Mouton de Gruyter. (= Studies in Generative Grammar 71).

Alexiadou, Artemis/Rathert, Monika (eds.) (2010): The semantics of nominalizations across languages and frameworks. Berlin/New York: de Gruyter.

Alexiadou, Artemis (2013): "Nominal vs. Verbal -ing Constructions and the Development of the English Progressive". English Linguistics Research 2/2: 126-140.

Alexiadou, Artemis/Iordăchioaia, Gianina (2015): "Idiomaticity and compositionality in deverbal compounds". Handout zum Vortrag auf der Konferenz The 8th Brussels Conference on Generative Linguistics: The Grammar of Idioms (BCGL8), Brüssel, 4.-5. Juni 2015.

Augst, Gerhard (1975): „Über das Fugenmorphem bei Zusammensetzungen“. In: Augst, Gerhard (ed.): Untersuchungen zum Morpheminventar der deutschen Gegenwartssprache. Tübingen, Narr: 71-155. (=Forschungsberichte des Instituts für deutsche Sprache 25).

Balles, Irene (2004): „Individuativa-Kontinuativa-Unterscheidung im Indogermanischen“. In: Fritz, Matthias/Wischer, Ilse (eds.): Historisch-Vergleichende Sprachwissenschaft und germanische Sprachen. Akten der 4. Neulandtagung der Historisch-Vergleichenden Sprachwissenschaft in Potsdam 2001. Budapest, Amulett Druck: 9-34. (=Innsbrucker Beiträge zur Sprachwissenschaft 113).

Bauer, Laurie (2001): Morphological productivity. Cambridge: Cambridge University Press.

Behaghel, Otto (1923/1932): Deutsche Syntax. Eine geschichtliche Darstellung. Band I und IV. Heidelberg: Winter.

Bloomer, Robert (1996): „Die pleonastischen Zusammensetzungen der deutschen Gegenwartssprache“. American Journal of Germanic Linguistics and Literature 8: 69-90.

Bücking, Sebastian (2012): „Müdigkeit und Müde-Sein: Zur Semantik adjektivbasierter Zustandsnominalisierungen“. Linguistische Berichte 232: 361-397.

Carr, Charles (1939): Nominal Compounds in Germanic. Oxford: Oxford University Press.

Cutler, Anne/Hawkings, John A./Gilligan, Gary (1985): “The suffixing preference: a processing explanation". Linguistics 23: 723-758.

Demske, Ulrike (2000): „Zur Geschichte der -ung-Nominalisierungen im Deutschen. Ein Wandel morphologischer Produktivität". PBB 122: 365-411. 
Demske, Ulrike (2001): Merkmale und Relationen. Diachrone Studien zur Nominalphrase des Deutschen. Berlin/New York: de Gruyter.

Dressler, Wolfgang U. (1968): Studien zur verbalen Pluralität. Iterativum, Distributivum, Durativum, Intensivum in der allgemeinen Grammatik, im Lateinischen und Hethitischen. Wien: Böhlau. (= Österreichische Akademie der Wissenschaften, Philosophischhistorische Klasse, Sitzungsberichte 259/1).

Dressler, Wolfgang/Lettner, Laura/Korecky-Kröll, Katharina (2010): "First language acquisition of compounds: with special emphasis on early German child language". In: Scalise, Sergio/Vogel, Irene (eds.): Cross-Disciplinary issues in compounding. Amsterdam: Benjamins: $323-344$.

Duden (2009): Die Grammatik. Duden Band 4. 8., neu erarbeitete und erweiterte Auflage. Herausgegeben von der Dudenredaktion. Mannheim/Zürich: Duden.

Ehrich, Veronika (1991): „Nominalisierungen“. In: Stechow, Arnim von/Wunderlich, Dieter (eds.): Semantik/Semantics. Ein internationales Handbuch der zeitgenössischen Forschung. An International Handbook of Contemporary Research. Berlin/New York, de Gruyter: 441-458. (= Handbücher zur Sprach- und Kommunikationswissenschaft/Handbooks of Linguistics and Communication Science 6).

Fuhrhop, Nanna (2000): „Zeigen Fugenelemente die Morphologisierung von Komposita an?““. In: Thieroff, Rolf et al. (eds.): Deutsche Grammatik in Theorie und Praxis. Tübingen: Niemeyer.

Gaeta, Livio (2008): “Constituent order in compounds and syntax: typology and diachrony". Morphology 18: 117-141.

Gaeta, Livio/Schlücker, Barbara (eds.) (2012): Das Deutsche als kompositionsfreudige Sprache. Strukturelle Eigenschaften und systembezogene Aspekte. Berlin/New York: de Gruyter. (= Linguistik - Impulse und Tendenzen 46).

Givón, Talmy (1971): "Historical syntax and synchronic morphology: an archaeologist's field trip". Chicago Linguistic Society 7/1: 394-415.

Grimm, Jacob (1837): Deutsche Grammatik. Band IV. Göttingen: Dieterichsche Buchhandlung.

Grimshaw, Jane (1990): Argument Structure. Cambridge, Mass.: MIT Press.

Gröger, Otto (1911): Die althochdeutsche und altsächsische Kompositionsfuge mit Verzeichnis der althochdeutschen und altsächsischen Composita. Zürich: Zürcher \& Furrer.

Haider, Hubert (2001): "Why are there no Complex Head-Initial Compounds?”. In: SchanerWolles, Chris/Rennison, John/Neubart, Friedrich (eds.): Naturally! Linguistic studies in honour of Wolfgang Ulrich Dressler presented on the occasion of his 60th birthday. Turin, Rosenberg \& Sellier: 165-174.

Haider, Hubert (2015): "Head directionality - in syntax and morphology". In: Fábregas, Antonio/Mateu, Jaume/Putnam, Mike (eds.): The Handbook of Parameters. London, Bloomsbury Academic: 73-97.

Härtl, Holden (2015): „Situationsargumente von Nicht-Köpfen: Verb-Nomen-Komposita im Zusammenspiel von Morphologie, Syntax und Pragmatik“. In: Fortmann, Christian/Lübbe, Anja/Rapp, Irene (eds.): Situationsargumente im Nominalbereich. Berlin/Boston: de Gruyter: 159-184. (= Linguistische Arbeiten 562).

Henzen, Walter (1965): Deutsche Wortbildung. 3. durchgesehene und ergänzte Auflage. Tübingen: Niemeyer. 
Heringer, Hans Jürgen (1995): „Prinzipien der Genuszuweisung“. In: Popp, Heidrun (ed.): Deutsch als Fremdsprache. An den Quellen eines Faches. Festschrift für Gerhard Helbig zum 65. Geburtstag. München, Iudicium: 203-216.

Heusinger, Klaus von (2010): „Zur Grammatik indefiniter Eigennamen“. Zeitschrift für germanistische Linguistik 38/2: 88-120.

Kastovsky, Dieter (2007): "Middle English word-formation. A list of desiderata". In: Mazzon, Gabriella (ed.): Studies in Middle English. Forms and Meanings. Frankfurt, Peter Lang: 41-56.

Kastovsky, Dieter (2012): "Linguistic Levels: Morphology". In: Bergs, Alexander/Brinton, Laurel (eds.): English Historical Linguistics. An International Handbook. Berlin/Boston, de Gruyter: 129-147. (= Handbücher zur Sprach- und Kommunikationswissenschaft/Handbooks of Linguistics and Communication Science 34/1).

Köpcke, Klaus-Michael/Zubin, David (2005): „Nominalphrasen ohne lexikalischen Kopf Zur Bedeutung des Genus für die Organisation des mentalen Lexikons am Beispiel der Autobezeichnungen im Deutschen“. Zeitschrift für Sprachwissenschaft 24: 93-122.

Korecky-Kröll, Katharina (2011): Der Erwerb der Nominalmorphologie bei zwei Wiener Kindern: Eine Untersuchung im Rahmen der Natürlichkeitstheorie. Dissertation, Universität Wien.

Krott, Andrea et al. (2007): “Analogical effects on linking elements in German compound words". Language and cognitive processes 22: 25-57.

Lehmann, Christian (1995): Thoughts on Grammaticalization. München/Newcastle: Lincom.

Lehmann, Christian (2005): „Wortarten und Grammatikalisierung“. In: Knobloch, Clemens/Schaeder, Burkhard (eds.): Wortarten und Grammatikalisierung. Perspektiven in System und Erwerb. Berlin/New York, de Gruyter: 1-20.

Leiss, Elisabeth (1992): Die Verbalkategorien des Deutschen. Ein Beitrag zur Theorie der sprachlichen Kategorisierung. Berlin/New York: de Gruyter. (= Studia Linguistica Germanica 31).

Leiss, Elisabeth (2005): „Derivation als Grammatikalisierungsbrücke für den Aufbau von Genusdifferenzierungen im Deutschen“. In: Leuschner, Torsten et al. (eds.): Grammatikalisierung im Deutschen. Berlin/New York, de Gruyter: 11-30.

Lexer, Matthias (1872-1878/2011): Mittelhochdeutsches Wörterbuch. 3 Bände. http://woerterbuchnetz.de/Lexer/ [06.02.2015].

Löbel, Elisabeth (1986): Apposition und Komposition in der Quantifizierung: Syntaktische, semantische und morphologische Aspekte quantifizierender Nomina im Deutschen. Tübingen: Niemeyer. (= Linguistische Arbeiten 166).

McIntyre, Andrew (2015): Deriving noun-incorporation when verbs don't normally nounincorporate: Argument-realising compounds in English and German. Handout zum Vortrag vom 23.01.2015, im Rahmen des Workshops „On Compounds“, Institut für Anglistik, Universität Stuttgart, im Rahmen des SFB 732 Incremental specification in context.

Neef, Martin/Borgwaldt, Susanne (2012): „Fugenelemente in neu gebildeten Nominalkomposita“. In: Gaeta, Livio/Schlücker, Barbara (eds.): Das Deutsche als kompositionsfreudige Sprache. Strukturelle Eigenschaften und systembezogene Aspekte. Berlin/New York, de Gruyter: 27-56. (= Linguistik - Impulse und Tendenzen 46).

Nübling, Damaris/Szczepaniak, Renata (2008): “On the way from morphology to phonology: German linking elements and the role of the phonological word". Morphology 18: 1-25. 
Nübling, Damaris/Szczepaniak, Renata (2010): „Was erklärt die Diachronie für die Synchronie der deutschen Gegenwartssprache? Am Beispiel schwankender Fugenelemente“. In: Schmid, Hans Ulrich (ed.): Perspektiven der germanistischen Sprachgeschichtsforschung. Berlin/New York, de Gruyter: 205-224. (= Jahrbuch für germanistische Sprachgeschichte 1).

Olsen, Susan (2012): „Der Einfluss des Mentalen Lexikons auf die Interpretation von Komposita“. In: Gaeta, Livio/Schlücker, Barbara (eds.): Das Deutsche als kompositionsfreudige Sprache. Strukturelle Eigenschaften und systembezogene Aspekte. Berlin/New York, de Gruyter: 135-170. (= Linguistik - Impulse und Tendenzen 46).

Osthoff, Hermann (1878): Das Verbum in der Nominalcomposition im Deutschen, Griechischen, Slavischen und Romanischen. Jena: Costenoble.

Paul, Hermann (1881/2007): Mittelhochdeutsche Grammatik. 25. Auflage, neu bearbeitet von Thomas Klein, Hans-Joachim Solms und Klaus-Peter Wegera. Mit einer Syntax von Ingeborg Schröbler, neubearbeitet und erweitert von Heinz-Peter Prell. Tübingen: Niemeyer.

Pavlov, Vladimir (2009): Deutsche Wortbildung im Spannungsfeld zwischen Lexikon und Syntax. Frankfurt etc.: Lang.

Pounder, Amanda (2001): "Adverb-marking in German and English. System and standardization”. Diachronica XVIII/2: 301-358.

Prell, Heinz-Peter (2000): „Die Stellung des attributiven Genitivs im Mittelhochdeutschen. Zur Notwendigkeit einer Syntax mittelhochdeutscher Prosa“". PBB 122: 23-39.

Rijkhoff, Jan (1992): The Noun Phrase. A typological Study of its Form and Structure. Amsterdam: Drukkerij Universiteit van Amsterdam.

Rivet, Anne (1999): „Rektionskomposita und Inkorporationstheorie“. Linguistische Berichte 179: 307-342.

Schlücker, Barbara (2012): „Die deutsche Kompositionsfreudigkeit. Übersicht und Einführung“'. In: Gaeta, Livio/Schlücker, Barbara (eds.): Das Deutsche als kompositionsfreudige Sprache. Strukturelle Eigenschaften und systembezogene Aspekte. Berlin/New York, de Gruyter: 1-27. (=Linguistik - Impulse und Tendenzen 46).

Schlücker, Barbara (2014): Grammatik im Lexikon. Adjektiv-Nomen-Verbindungen im Deutschen und Niederländischen. Berlin/Boston: de Gruyter. (=Linguistische Arbeiten 553).

Schuster, Saskia (2016): Variation und Wandel. Zur Konkurrenz morphologischer und syntaktischer A+N-Verbindungen im Deutschen und Niederländischen seit 1700. Berlin: Mouton de Gruyter. (= Konvergenz und Divergenz 4$)$.

Seiler, Hansjakob/Lehmann, Christian (eds.) (1982): Apprehension. Das sprachliche Erfassen von Gegenständen. Teil 1: Bereich und Ordnung der Phänomene. Tübingen: Narr.

Solling, Daniel (2012): Zur Getrennt-, Zusammen- und Bindestrichschreibung von Substantivkomposita im Deutschen (1550-1710). Uppsala: Universität Uppsala. (= Acta Universitatis Upsaliensis. Studia Germanistica Upsaliensia 57).

Splett, Jochen (1993): Althochdeutsches Wörterbuch. Analyse der Wortfamilienstrukturen des Althochdeutschen, zugleich Grundlegung einer Strukturgeschichte des deutschen Wortschatzes. 3 Bände. Berlin/New York: de Gruyter.

Splett, Jochen (2000): „Wortbildung des Althochdeutschen.“ In: Besch, Werner/Betten, Anne/Reichmann, Oskar/Sonderegger, Stefan (eds.): Sprachgeschichte. 2. Teilband. 2. Aufl. Berlin/New York, de Gruyter: 1213-1221. (= Handbücher zur Sprach- und Kommunikationswissenschaft/Handbooks of Linguistivs and Communication Science 2). 
Steinbach, Markus (2002): Middle voice. A comparative study in the syntax-semantics interface of German. Amsterdam: Benjamins. (=Linguistik Aktuell/Linguistics Today 50).

Vogel, Petra M. (1996): Wortarten und Wortartenwechsel. Zu Konversion und verwandten Erscheinungen im Deutschen und in anderen Sprachen. Berlin/New York: de Gruyter. (= Studia Linguistica Germanica 7).

Wanzeck, Christiane (2003): Die Kompositabildung im Frühneuhochdeutschen. Eine Studie zu den Entwicklungstendenzen und deren Faktoren. Unveröffentlichte Habilitationsschrift LMU München.

Wegener, Heide (2005): „Das Hühnerei vor der Hundehütte. Von der Notwendigkeit historischen Wissens in der Grammatikographie des Deutschen“. In: Berner, Elisabeth/Böhm, Manuela/Voeste, Anja (eds.): ein gross und narrhafft haffen. Festschrift für Joachim Gessinger. Potsdam, Universitäts-Verlag: 176-187.

Werner, Martina (2010): „Substantivierter Infinitiv statt Derivation. Ein ,echter' Genuswechsel und ein Wechsel der Kodierungstechnik innerhalb der deutschen Verbalabstraktbildung“. In: Bittner, Dagmar/Gaeta, Livio (eds.): Kodierungstechniken im Wandel. Das Zusammenspiel von Analytik und Synthese im Gegenwartsdeutschen. Berlin/New York, de Gruyter: 159-178. (= Linguistik-Impulse \& Tendenzen 34).

Werner, Martina (2012): Genus, Derivation und Quantifikation. Zur Funktion der Suffigierung und verwandter Phänomene im Deutschen. Berlin/Boston: de Gruyter. (= Studia Linguistica Germanica 114).

Werner, Martina (2016): „Genus und Fugenelemente. Zur Herleitung einer motivierten Relation“. In: Ernst, Peter/Werner, Martina (eds.): Linguistische Pragmatik in historischen Bezügen. Berlin/Boston: de Gruyter. (= Lingua Historica Germanica 9).

Wiese, Richard (1996): "Phrasal Compounds and the Theory of Word Syntax". Linguistic Inquiry 27/1: 183-193.

Wilmanns, Wilhelm (1893): Deutsche Grammatik. Lautlehre. Band 1. Straßburg: Trübner. Wilmanns, Wilhelm (1896): Deutsche Grammatik. Wortbildung. Band 2. Straßburg: Trübner. Wurzel, Wolfgang (1996): „Morphologischer Strukturwandel: Typologische Entwicklungen im Deutschen“. In: Lang, Ewald/Zifonun, Gisela (eds.): Deutsch - typologisch. Berlin/New York, de Gruyter: 492-524.

Zifonun, Gisela (2010): „Von Bush administration zu Kohl-Regierung: Englische Einflüsse auf deutsche Nominalkonstruktionen?“. In: Scherer, Carmen/Holler, Anke (eds.): Strategien der Integration und Isolation nicht-nativer Einheiten und Strukturen. Berlin/New York, de Gruyter: 165-182. (= Linguistische Arbeiten 532).

Zifonun, Gisela (2012): „Komposition (oder Halbaffigierung) zum Ausdruck von Nominalaspekt: Schmuckstück, Glücksfall und Zuckerwerk“. In: Gaeta, Livio/Schlücker, Barbara (eds.): Das Deutsche als kompositionsfreudige Sprache. Strukturelle Eigenschaften und systembezogene Aspekte. Berlin/New York, de Gruyter: 101-134. (= Linguistik - Impulse und Tendenzen 46). 